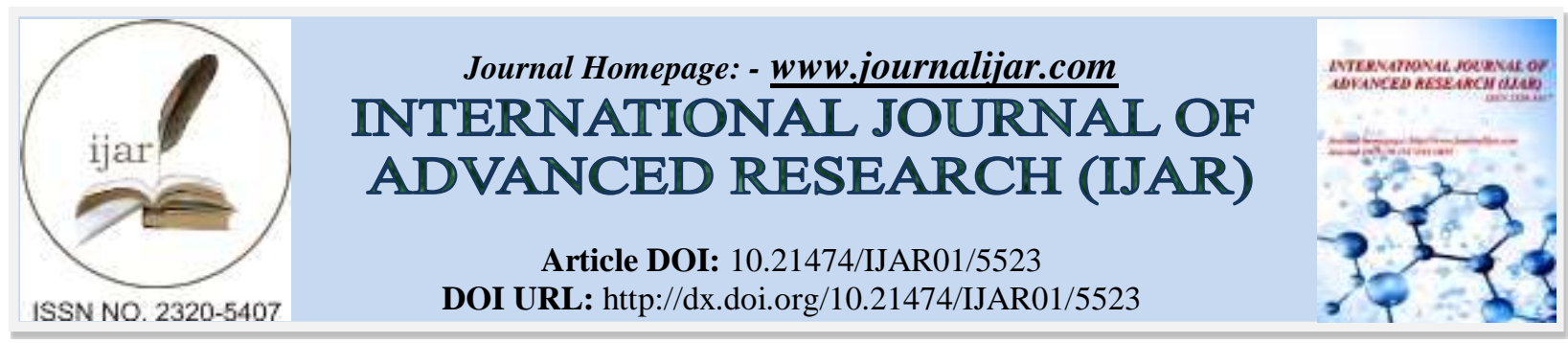

RESEARCH ARTICLE

\title{
STABILITY ANALYSIS OF NONLINEAR CONTROL USING HIERARCHICAL DYNAMIC INVERSION FOR A WINGED ROCKET.
}

\author{
"Hiroshi Yamasaki, Koichi Yonemoto and Takahiro Fujikawa. \\ Department Of Mechanical And Control Engineering, Kyushu Institute Of Technology, Japan.
}

\section{Manuscript Info}

Manuscript History

Received: 03 August 2017

Final Accepted: 05 September 2017

Published: October 2017

Key words:-

Stability Analysis, Nonlinear System,

Autonomous Control,

Dynamic Inversion, Winged Rocket.

\section{Abstract}

This paper presents stability analysis and time response evaluation of nonlinear control based on the hierarchy Dynamic Inversion (DI) and the block strict-feedback form, assuming the application to a winged rocket vehicle. Such vehicles have a wide range of flight conditions, and the associated change in aerodynamic characteristics during the flight leads to highly nonlinear dynamics. Whereas DI theory can cancel the nonlinear dynamics and linearize the input and output maps, it becomes troublesome to construct the control law when a system has high relative degree between the input and output maps. DI theory combined with time-scale separation, on the other hand, can provide a simple control law at the expense of strict linearization, and it has been one of the effective control methods for nonlinear system. However, there is a difficulty in evaluating the stability of such a control law. In order to solve these problems, the hierarchical DI method in the block strict-feedback form is investigated in this paper. This methodology is advantageous in that the control law is simple and its stability can be analyzed via classical eigenvalue analysis. The developed technique is applied to an experimental winged rocket vehicle whose dynamics consist of hierarchical structures of vehicle dynamics and actuator dynamics, and its validity is demonstrated via numerical simulations.

Copy Right, IJAR, 2017,. All rights reserved.

\section{Introduction:-}

In recent years, reusable launch vehicles have been researched and developed around the world in order to reduce the space transportation cost and to facilitate the space development. Among possible concepts of future space transportation system, s winged-type vehicle is advantageous from its reusability, operability, and abort capability. The dynamics of such a vehicle is highly nonlinear, because the aerodynamic characteristics dynamically change along its flight profile. For example, in suborbital flight, flight sequence contains powered-ascent, coasting, apogee flight, reentry, gliding, and landing, where dynamic pressure and Mach number undergo drastic changes. Therefore, there is an increasing demand in developing nonlinear control methodologies for future space transportation system.

This paper focuses on the development of dynamic inversion (DI) theory for nonlinear control. DI theory is a control technique that cancels nonlinear dynamics of the system and realizes desirable dynamics via algebraic transformation $^{1-3)}$. Using DI theory, however, it becomes troublesome to construct and implement the control law 
when the system has high relative degree between the input and output maps, because the lengthy derivative calculation is required. In order to overcome this difficulty, studies on flight control based on DI theory combined with time-scale separation were initially conducted by Menon et al. ${ }^{4}$ ), and these works are referred by many succeeding researches ${ }^{8-14)}$. Time-scale separation is a concept that stems from the singular perturbation theory, and it enables to construct the hierarchical dynamics when the vehicle dynamics can be divided into subsystems with explicitly different time scales ${ }^{15}$. Different time constants inside a system can exist corresponding to, for example, translational vehicle motions, rotational vehicle motions, and actuator dynamics. When the control law is properly designed and applied to each subsystem, and the resulting time-scale is sufficiently separated, the hierarchical structure can greatly simplify the design of the control law and its analysis. Outer loop of the time-scale separated system has relatively large time constant, and it is called "slow-scale". On the other hand, inner loop has small time constant, and it is called "fast-scale". As a rule of thumb, it is generally considered that the ratio of these time constants has to be more than three for the sufficient separation ${ }^{9)}$.

However, the control law of slow-scale subsystem using the time-scale separation approach is designed with the assumption that the dynamic variable with fast-scale is always on an equilibrium state. This means that, dynamics interference between the subsystems is neglected, and it is stated by Abe, et al. that it is difficult to guarantee the stability of such a closed-loop system ${ }^{16}$. In addition, design procedures of the controller are somewhat empirical, and they require experience.

Abe et al. developed an adaptive control law that guarantees Lyapunov stability using backstepping method ${ }^{16-17)}$. Generally, the derivative of intermediate controlled variable must be prepared, when the control law is constructed using backstepping method ${ }^{18)}$. This makes the analysis even more difficult, and the control law becomes complicated. Abe et al. ${ }^{16-17)}$ circumvented this problem by estimating the derivative of intermediate controlled variable via an adaptive algorithm instead of calculating it exactly. This can prevent the control system being too complicated. The validity of the method was demonstrated via carrying out numerical simulation using ALFLEX (Automatic Landing Flight Experimental) vehicle as a model, and the result indicated that the method can improve the robustness against disturbances in the lateral direction. However, the methodology for determining control gains such that realize Lyapunov stability is not completely established.

So as to solve the foregoing problems, a novel kind of hierarchical dynamic inversion based on the block-strict feedback form of backstepping method is investigated in this paper. Its basic idea is to construct a transfer function between the state and pseudo input for each hierarchy and to ignore its nonlinear term. Since the dynamic characteristics of lower hierarchy subsystem affect the higher hierarchy subsystem in reality, the nonlinear dynamic characteristics of higher hierarchy cannot be cancelled completely using the proposed approach. On the contrary, when the response of the lower subsystem is sufficiently faster than that of the higher subsystem, the error resulting from this linear approximation becomes small. As a consequence, linear dynamic characteristics of the higher subsystem that is not affected by the nonlinear dynamic characteristics of lower subsystem can be evaluated. This idea is similar to that of the forced singular perturbation theory ${ }^{13)}$. There are mainly two advantages in the developed methodology. First, the process to design the control gains from the characteristics of the closed-loop dynamics is further simplified. Second, the effectiveness of the gain design methodology can be verified via performing eigenvalue-based stability analysis. In order to assess the influence of the linear approximation, the instantaneous linearization of the nonlinear dynamics is constructed as well, and the difference between their eigenvalues is examined in this paper.

Vehicle model utilized in this paper is that of WIRES\#015 (WInged REusable Sounding rocket), which is an experimental winged rocket developed in Kyushu Institute of Technology ${ }^{19-23)}$. Figure 1 and Table 1 show the overview and specifications of WIRES\#015, respectively. Among the flight phases of WIRES\#015, stability analysis during the gliding phase is conducted. 


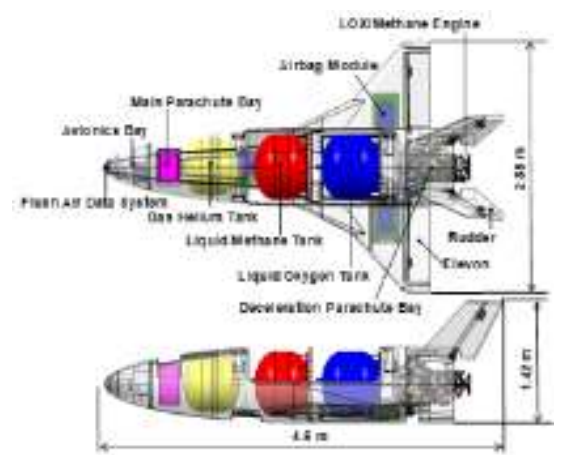

Figure 1:- Overview of WIRES\#015
Table 1:- Specifications of WIRES\#015

\begin{tabular}{|l|l|l|}
\hline Vehicle dry mass & {$[\mathrm{kg}]$} & 672 \\
\hline Body Length & {$[\mathrm{m}]$} & 4 \\
\hline Wing area & {$\left[\mathrm{m}^{2}\right]$} & 2.68 \\
\hline Wing span & {$[\mathrm{m}]$} & 2.88 \\
\hline $\begin{array}{l}\text { Mean aerodynamic } \\
\text { chord }\end{array}$ & {$[\mathrm{m}]$} & 1.08 \\
\hline Moment of inertia & {$\left[\mathrm{kg} \mathrm{m}^{2}\right]$} & 1138 \\
\hline Center of gravity & {$[\%]$} & 66 \\
\hline
\end{tabular}

\section{Hierarchal Dynamic Inversion Methodology:- \\ Dynamic Inversion Theory}

Dynamic inversion theory is a methodology for linearizing nonlinear dynamics between input and output maps. In this section, the DI methodology is described briefly ${ }^{1)}$. For the sake of simplicity, a nonlinear affine system with single input and single output expressed as eqs. (1) and (2) is considered.

$$
\begin{aligned}
\dot{\mathbf{x}} & =f(\mathbf{x})+g(\mathbf{x}) u \\
y & =h(\mathbf{x})
\end{aligned}
$$

where

$$
\mathbf{x}=\left(x_{1}, x_{2}, \ldots, x_{n}\right)
$$

is the $n$-dimension state variable of system, $y$ is the controlled variable, and $u$ is the manipulated variable. $f(\mathbf{x})$, $g(\mathbf{x})$, and $h(\mathbf{x})$ are the functions of $\mathbf{x}$. These mappings are represented in the form of $n$-dimensional vectors of real-valued functions of the real variable $\mathbf{x}$, namely

$$
\begin{aligned}
& f(x)=\left[\begin{array}{c}
f_{1}(\mathbf{x}) \\
f_{2}(\mathbf{x}) \\
\vdots \\
f_{n}(\mathbf{x})
\end{array}\right] \\
& g(\mathbf{x})=\left[\begin{array}{c}
g_{1}(\mathbf{x}) \\
g_{2}(\mathbf{x}) \\
\vdots \\
g_{n}(\mathbf{x})
\end{array}\right] \\
& h(\mathbf{x})=h_{1}(\mathbf{x})
\end{aligned}
$$

Let $L_{f}$ be the Lie derivative whose definition is presented in the following eq. ( 7 ).

$$
L_{f} h=\frac{d h}{d x} f=\sum_{i=1}^{n} \frac{\partial h}{\partial x_{i}} f_{i}
$$

In addition, the $(i+1)$ th order Lie derivative $L^{i+1} f h$ is defined as

$$
L^{i+1}{ }_{f} h=L_{f}\left(L_{f} h\right), i=1,2, \ldots
$$

The value of $r$ such that satisfies eq. ( 9 ) is referred to as the relative degree of the system.

$$
L_{g} L^{r-1} h=\sum_{i=1}^{n} \frac{\partial L^{r-1} h h}{\partial x_{i}} g_{i} \neq 0
$$

When the relative degree of the system is $r$, the $r$ th order derivative of controlled variable $y$ is expressed by eq. ( 10 ) below.

$$
y^{(r)}=L_{f} h+\left(L_{g} L^{r-1} h\right) u
$$

From eq. ( 10 ), an manipulated variable $u$ can be calculated from the $r$ th order derivative of controlled variable by

$$
u=\left(L_{g} L_{f}^{r-1} h\right)^{-1}\left(y^{(r)}-L_{f}^{r} h\right)
$$

which expresses inverse dynamics of manipulated variable $u$. Then, by introducing the pseudo input $v$ defined by 


$$
y^{(r)}=v
$$

control command can be generated using eq. ( 13 ) below.

$$
u_{\text {com }}=\left(L_{g} L_{f}^{r-1} h\right)^{-1}\left(v-L_{f}^{r} h\right)
$$

Since $v$ can be designed in an arbitrary manner, the linear feedback law shown in eq. (14) is utilized for realizing linear response in this paper.

$$
v=-\left(a_{r-1} y^{(r-1)}+a_{r-2} y^{(r-2)}+\cdots+a_{0} y\right)+a_{0} y_{\text {com }}
$$

Using eq. ( 13 ) and eq. ( 14 ), the transfer function of the nonlinear system based on DI is obtained as follows.

$$
P(s)=\frac{y}{y_{\mathrm{com}}}=\frac{a_{0}}{s^{r}+a_{r-1} s^{r-1}+\cdots+a_{0}}
$$

\section{Hierarchal Dynamic Inversion:-}

Using DI, a nonlinear system can be handled like a linear system. However, as the relative degree of a nonlinear system increases, the higher-order Lie derivatives appeared in eq. ( 10 ) contain the increasing number of terms, and the control law becomes more complicated. In this paper, in order to mitigate this problem, the nonlinear system is expressed by block-strict-feedback form consisting of hierarchal $m$ subsystems as follows instead of affine system used in previous researches.

$$
\begin{aligned}
& {\left[\begin{array}{l}
\dot{\mathbf{x}}_{1} \\
\dot{\mathbf{x}}_{2} \\
\vdots \\
\dot{\mathbf{x}}_{i} \\
\vdots \\
\dot{\mathbf{x}}_{m-1} \\
\dot{\mathbf{x}}_{m}
\end{array}\right]=\left[\begin{array}{c}
F_{1}\left(\mathbf{x}_{1}\right) \\
F_{2}\left(\mathbf{x}_{1}, \mathbf{x}_{2}\right) \\
\vdots \\
F_{i}\left(\mathbf{x}_{1}, \ldots, \mathbf{x}_{i}\right) \\
\vdots \\
F_{m-1}\left(\mathbf{x}_{1}, \ldots, \mathbf{x}_{m-1}\right) \\
F_{m}\left(\mathbf{x}_{1}, \ldots, \mathbf{x}_{m}\right)
\end{array}\right]+\left[\begin{array}{c}
G_{1}\left(\mathbf{x}_{1}\right) y_{2} \\
G_{2}\left(\mathbf{x}_{1}, \mathbf{x}_{2}\right) y_{3} \\
\vdots \\
G_{i}\left(\mathbf{x}_{1}, \ldots, \mathbf{x}_{i}\right) y_{i+1} \\
\vdots \\
y_{m-1}\left(\mathbf{x}_{1}, \ldots, \mathbf{x}_{m-1}\right) y_{m} \\
G_{m}\left(\mathbf{x}_{1}, \ldots, \mathbf{x}_{m}\right) u
\end{array}\right]} \\
& {\left[\begin{array}{c}
y_{1} \\
y_{2} \\
\vdots \\
y_{i} \\
\vdots \\
y_{m-1} \\
y_{m}
\end{array}\right]=\left[\begin{array}{c}
\left.\mathbf{x}_{1}\right) \\
h_{2}\left(\mathbf{x}_{2}\right) \\
\vdots \\
h_{i}\left(\mathbf{x}_{i}\right) \\
\vdots \\
h_{m-1}\left(\mathbf{x}_{m-1}\right) \\
h_{m}\left(\mathbf{x}_{m}\right)
\end{array}\right]}
\end{aligned}
$$

In the $i$ th subsystem, the state and output belong to $\mathbf{x}_{i} \in \mathfrak{R}^{n_{i}}$ and $y_{i} \in \mathfrak{R}$, respectively, and the $F_{i}$ and $G_{i}$ are functions with the dimension of $n_{i}$.

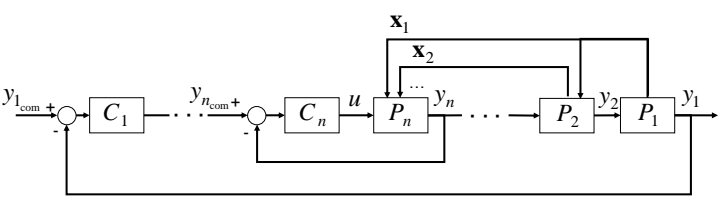

(a) Diagram of overall system

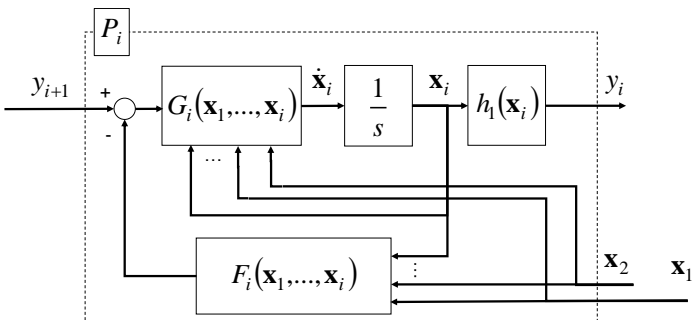

(b) Diagram of each subsystem

Figure 2. Block diagrams of a system in block-strict feedback form with controller And also, equations of each subsystem of block-strict feedback form are eqs. ( 18 ), and ( 19 ). $F_{i}\left(\mathbf{x}_{1}, \cdots, \mathbf{x}_{i}\right)$ and $G_{i}\left(\mathbf{x}_{1}, \cdots, \mathbf{x}_{i}\right)$ denote the values at a specific point $\mathbf{x}_{1}, \cdots, \mathbf{x}_{i}$. These mappings are represented in the form of $n_{i}$ dimensional vectors of real-valued functions of the real variables $\mathbf{x}_{1}, \cdots, \mathbf{x}_{i}$, namely

$$
F_{i}\left(\mathbf{x}_{1}, \cdots, \mathbf{x}_{i}\right)=\left[\begin{array}{c}
f_{i, 1}\left(\mathbf{x}_{1}, \cdots, \mathbf{x}_{i}\right) \\
f_{i, 2}\left(\mathbf{x}_{1}, \cdots, \mathbf{x}_{i}\right) \\
\vdots \\
f_{i, n_{i}}\left(\mathbf{x}_{1}, \cdots, \mathbf{x}_{i}\right)
\end{array}\right], i=1,2, \ldots, m
$$




$$
G_{i}\left(\mathbf{x}_{1}, \cdots, \mathbf{x}_{i}\right)=\left[\begin{array}{c}
g_{i, 1}\left(\mathbf{x}_{1}, \cdots, \mathbf{x}_{i}\right) \\
g_{i, 2}\left(\mathbf{x}_{1}, \cdots, \mathbf{x}_{i}\right) \\
\vdots \\
g_{i, n_{i}}\left(\mathbf{x}_{1}, \cdots, \mathbf{x}_{i}\right)
\end{array}\right], i=1,2, \ldots, m
$$

The relative degree of the $i$ th hierarchy $r_{i}$ is defined as follows.

$$
L_{G_{i}} L^{r_{i}-1} F_{i} h_{i}\left(\mathbf{x}_{i}\right) \neq 0, i=1,2, \ldots, m
$$

It is noted that the relation of $r_{i} \leq n_{i}$ holds. From eqs.( 16 ), ( 17 ), and ( 20 ) and the DI method explained in the previous section, command in each hierarchy is obtained by the following equation.

$$
\left\{\begin{aligned}
y_{2_{\text {com }}} & =\left[L_{G_{1}} L_{F_{1}}{ }^{r_{1}-1} h_{1}\left(\mathbf{x}_{1}\right)\right]^{-1}\left[v_{1}-L_{F_{1}}{ }^{r_{1}} h_{1}\left(\mathbf{x}_{1}\right)\right] \\
y_{3_{\mathrm{com}}} & =\left[L_{G_{2}} L_{F_{2}}{ }^{r_{2}-1} h_{2}\left(\mathbf{x}_{2}\right)\right]^{-1}\left[v_{2}-L_{F_{2}}{ }^{r_{2}} h_{2}\left(\mathbf{x}_{2}\right)\right] \\
& \vdots \\
y_{i+1_{\mathrm{com}}} & =\left[L_{G_{i}} L_{F_{i}}{ }^{r_{i}-1} h_{i}\left(\mathbf{x}_{i}\right)\right]^{-1}\left[v_{i}-L_{F_{i}}{ }^{r_{i}} h_{i}\left(\mathbf{x}_{i}\right)\right] \\
& \vdots \\
y_{m_{\mathrm{com}}} & =\left[L_{G_{m-1}} L_{F_{m-1}}{ }^{r_{m-1}-1} h_{m-1}\left(\mathbf{x}_{m-1}\right)\right]^{-1}\left[v_{m-1}-L_{F_{m-1}}{ }^{r_{m-1}} h_{m-1}\left(\mathbf{x}_{m-1}\right)\right] \\
u_{\mathrm{com}} & =\left[L_{G_{m}} L_{F_{m}}{ }^{r_{m}-1} h_{m}\left(\mathbf{x}_{m}\right)\right]^{-1}\left[v_{m}-L_{F_{m}}{ }^{r_{m}} h_{m}\left(\mathbf{x}_{m}\right)\right]
\end{aligned}\right.
$$

where, $v_{i}(i=1,2, \ldots, m)$ is pseudo input in each hierarchy, and it is expressed by the following linear feedback law in order to realize linear response.

$$
\left\{\begin{aligned}
v_{1} & =-\left(a_{1, r_{1}-1} y_{1}{ }^{\left(r_{1}-1\right)}+\cdots+a_{1,0} y_{1}\right)+a_{1,0} y_{1_{\mathrm{com}}} \\
v_{2} & =-\left(a_{2, r_{2}-1} y_{2}{ }^{\left(r_{2}-1\right)}+\cdots+a_{2,0} y_{2}\right)+a_{2,0} y_{2_{\mathrm{com}}} \\
& \vdots \\
v_{i} & =-\left(a_{i, r_{i}-1} y_{i}{ }^{\left(r_{i}-1\right)}+\cdots+a_{i, 0} y_{i}\right)+a_{i, 0} y_{i_{\mathrm{com}}} \\
& \vdots \\
v_{m-1} & =-\left(a_{m-1, r_{m-1}-1} y_{m-1}{ }^{\left(r_{m-1}-1\right)}+\cdots+a_{m-1,0} y_{m-1}\right)+a_{m-1,0} y_{m-1_{\mathrm{com}}} \\
v_{m} & =-\left(a_{m, r_{m}-1} y_{m}{ }^{\left(r_{m}-1\right)}+\cdots+a_{m, 0} y_{m}\right)+a_{m, 0} y_{m_{\mathrm{com}}}
\end{aligned}\right.
$$

Here, $a_{i, r_{i}-j}\left(i=1, \ldots, m, j=1, \ldots, r_{j}\right)$ are the design parameters of the control law. By substituting eq. ( 22 ) into eq. ( 21 ), and eq. ( 21 ) into the $r_{m}$ th order derivatives of the controlled variable ( eq.( 17 )) in the $m$ th hierarchy is obtained as eq. ( 23$)-(25)$.

$$
\begin{aligned}
y_{m}{ }^{\left(r_{m}\right)} & =L_{F_{m}}{ }^{{ }^{r}} h\left(\mathbf{x}_{m}\right)+L_{G_{m}} L_{F_{m}}{ }^{r_{m}-1} h\left(\mathbf{x}_{m}\right) u_{\mathrm{com}} \\
& =v_{m} \\
& =-\left(a_{m, r_{m}-1} y_{m}{ }^{\left(r_{m}-1\right)}+\cdots+a_{m, 0} y_{m}\right)+a_{m, 0} y_{m_{\mathrm{com}}}
\end{aligned}
$$

Therefore, the transfer function of controlled variable in the $m$ th hierarchy is expressed as follows.

$$
P_{m}(s)=\frac{y_{m}}{y_{m_{\mathrm{com}}}}=\frac{a_{m, 0}}{s^{r_{m}}+a_{m, r_{m}-1} s^{r_{m}-1}+\cdots+a_{m, 0}}
$$

In addition, the $r_{m-1}$ th order derivative of the controlled variable in the $(m-1)$ th hierarchy is rewritten as follows utilizing eq. ( 21 ).

$$
\begin{aligned}
y_{m-1}{ }^{\left(r_{m-1}\right)} & =L_{F_{m-1}}{ }^{r_{m-1}} h_{m-1}\left(\mathbf{x}_{m-1}\right)+L_{G_{m-1}} L_{F_{m-1}}{ }^{r_{m-1}-1} h_{m-1}\left(\mathbf{x}_{m-1}\right) y_{m} \\
& =L_{F_{m-1}}{ }^{r_{m-1}} h_{m-1}\left(\mathbf{x}_{m-1}\right)+L_{G_{m-1}} L_{F_{m-1}}{ }^{r_{m-1}-1} h_{m-1}\left(\mathbf{x}_{m-1}\right) \frac{y_{m}}{y_{m_{\mathrm{com}}}} y_{m_{\mathrm{coom}}} \\
& =L_{F_{m-1}}{ }^{{ }_{m-1}} h_{m-1}\left(\mathbf{x}_{m-1}\right)\left[1-\frac{y_{m}}{y_{m_{\mathrm{com}}}}\right]+\frac{y_{m}}{y_{m_{\mathrm{com}}}} v_{m-1}
\end{aligned}
$$

The most right hand side of eq. ( 29 ) can be divided into a nonlinear term and a linear term in the following manner. 


$$
y_{m-1}^{\left(r_{m-1}\right)}=T F_{n l}+T F_{l},
$$

where

$$
\begin{aligned}
T F_{n l} & =L_{F_{m-1}} r_{m-1} h_{m-1}\left(\mathbf{x}_{m-1}\right)\left[1-\frac{y_{m}}{y_{m_{\mathrm{com}}}}\right] \\
T F_{l} & =\frac{y_{m}}{y_{m_{\mathrm{com}}}} v_{m-1}
\end{aligned}
$$

are the nonlinear term and the linear term, respectively. When the response of the controlled variable in the $m$ th hierarchy is sufficiently fast, $\left(y_{m} / y_{m \text { com }}\right)$ can be approximated to one, and the effect of the nonlinear term disappears. When the nonlinear term is ignored, eq. ( 27 ) turns out to be eq. ( 32 ).

$$
y_{m-1}{ }^{\left(r_{m-1}\right)} \cong \frac{y_{m}}{y_{m_{\text {com }}}} v_{m-1}
$$

By substituting eq. ( 22 ) and eq. ( 26 ) into eq. ( 32 ), the transfer function in the (m-1)th hierarchy is described as follows.

$$
P_{m-1}(s)=\frac{y_{m-1}}{y_{m-1_{\text {com }}}}=\frac{P_{m} a_{m-1,0}}{s_{m-1}^{r_{m-1}}+P_{m} a_{m-1, r_{m-1}-1} s^{r_{m-1}-1}+\cdots+P_{m} a_{m-1,0}}
$$

Transfer functions in higher hierarchies can be derived by repeating the procedures from eq. ( 27 ) to eq. ( 33 ), which gives

$$
\left\{\begin{aligned}
& P_{1}(s)=\frac{y_{1}}{y_{c o m}}=\frac{P_{2} a_{1,0}}{s^{r_{1}}+P_{2} a_{1, r_{1}-1} s^{r_{1}-1}+\cdots+P_{2} a_{1,0}} \\
& P_{2}(s)=\frac{y_{2}}{y_{2_{\mathrm{com}}}}=\frac{P_{3} a_{2,0}}{s^{r_{2}}+P_{3} a_{2, r_{2}-1} s^{r_{2}-1}+\cdots+P_{3} a_{2,0}} \\
& \vdots \\
& P_{i}(s) \quad \frac{y_{i}}{y_{i_{\mathrm{com}}}}=\frac{P_{i+1} a_{2,0}}{s^{r_{i}}+P_{i+1} a_{i, r_{i}-1} s^{r_{i}-1}+\cdots+P_{i+1} a_{i, 0}} \\
& \vdots \\
& P_{m-1}(s) \quad=\frac{y_{m-1}}{y_{m-1}}=\frac{P_{m} a_{m-1,0}}{s^{r_{m-1}+P_{m} a_{m-1, r_{m-1}-1} s^{r_{m-1}-1}+\cdots+P_{m} a_{m-1,0}}} \\
& P_{m}(s) \quad=\frac{y_{m}}{y_{m_{\mathrm{com}}}}=\frac{a_{m, 0}}{s^{r_{m}}+a_{m, r_{m}-1} s^{r_{m}-1}+\cdots+a_{m, 0}}
\end{aligned}\right.
$$

Hereafter, transfer functions presented in eq. ( 34 ) are called "Linearized Approximation Transfer Functions (LATF)".

\section{Application to Experimental Winged Rocket:-}

In order to evaluate the validity of LATF, the flight dynamics of WIRES\#015, an experimental winged rocket developed in Kyushu Institute of Technology, is utilized as a test case. The longitudinal dynamics during the unpowered gliding phase is considered. Dynamics of WIRES contains aerodynamic terms that are nonlinear with respect to flight conditions (e.g. velocity and aerodynamic attitudes). In addition to the translational and rotational vehicle dynamics, dynamics of the actuators for the aerodynamic control surfaces is considered.

\section{Plant Dynamics Model:-}

It is possible to construct several hierarchical dynamics models based on block-strict feedback form, because there is no rigorous criterion to determine the number of hierarchical subsystems and to divide the whole system into them. In this paper, the following two-level hierarchical structure is employed in order to assess the characteristics of the proposed method as simple as possible. The first hierarchy is the longitudinal vehicle dynamics, and the second hierarchy is the actuator dynamics expressed as the second-order delay model.

$$
\left[\begin{array}{c}
\dot{\mathbf{x}}_{1} \\
\dot{\mathbf{x}}_{2}
\end{array}\right]=\left[\begin{array}{c}
F_{1}\left(\mathbf{x}_{1}\right) \\
F_{2}\left(\mathbf{x}_{2}\right)
\end{array}\right]+\left[\begin{array}{l}
G_{1}\left(\mathbf{x}_{1}\right) y_{2} \\
G_{2}\left(\mathbf{x}_{2}\right) u
\end{array}\right]
$$




$$
\begin{aligned}
& {\left[\begin{array}{l}
y_{1} \\
y_{2}
\end{array}\right]=\left[\begin{array}{l}
h_{1}\left(\mathbf{x}_{1}\right) \\
h_{2}\left(\mathbf{x}_{2}\right)
\end{array}\right]=\left[\begin{array}{l}
\alpha \\
\delta_{e}
\end{array}\right]} \\
& \mathbf{x}_{1}=\left[\begin{array}{llll}
V_{c} & \alpha & Q & \Theta
\end{array}\right]^{T} \\
& \mathbf{x}_{2}=\left[\begin{array}{ll}
\delta_{e} & \dot{\delta}_{e}
\end{array}\right]^{T} \\
& u=\delta_{e_{\mathrm{cou}}} \\
& F_{1}\left(\mathbf{x}_{1}\right)=\left[\begin{array}{llll}
f_{1,1}\left(\mathbf{x}_{1}\right) & f_{1,2}\left(\mathbf{x}_{1}\right) & f_{1,3}\left(\mathbf{x}_{1}\right) & f_{1,4}\left(\mathbf{x}_{1}\right)
\end{array}\right]^{t} \\
& F_{2}\left(\mathbf{x}_{2}\right)=\left[\begin{array}{ll}
f_{2,1}\left(\mathbf{x}_{2}\right) & f_{2,2}\left(\mathbf{x}_{2}\right)
\end{array}\right]^{T} \\
& G_{1}\left(\mathbf{x}_{1}\right)=\left[\begin{array}{llll}
0 & 0 & g_{1,3}\left(\mathbf{x}_{1}\right) & 0
\end{array}\right]^{T} \\
& G_{2}\left(\mathbf{x}_{2}\right)=\left[\begin{array}{ll}
0 & g_{2,2}\left(\mathbf{x}_{2}\right)
\end{array}\right]^{T}
\end{aligned}
$$

where

$$
\begin{aligned}
& f_{1,1}\left(\mathbf{x}_{1}\right)=-g \sin (\Theta-\alpha)-\frac{\rho V_{c}^{2} S}{2 m} C_{D} \\
& f_{1,2}\left(\mathbf{x}_{1}\right)=Q+\frac{g}{V_{c}} \cos (\Theta-\alpha)-\frac{\rho V_{c} S}{2 m} C_{L} \\
& f_{1,3}\left(\mathbf{x}_{1}\right)=\frac{\rho V_{c}^{2} S \bar{c}}{2 I_{y y}}\left[C_{m}+\frac{\bar{c}}{2 V_{c}}\left(C_{m_{q}} \frac{180}{\pi} Q+C_{m_{\dot{\alpha}}} \frac{180}{\pi} \dot{\alpha}\right)\right] \\
& f_{1,4}\left(\mathbf{x}_{1}\right)=Q \\
& f_{2,1}\left(\mathbf{x}_{2}\right)=\dot{\delta}_{e} \\
& f_{2,2}\left(\mathbf{x}_{2}\right)=-\omega_{\delta_{e}}^{2} \delta_{e}-2 \zeta_{\delta_{e}} \omega_{\delta_{e}} \dot{\delta}_{e} \\
& g_{1,3}\left(\mathbf{x}_{1}\right)=\frac{\rho V_{c}^{2} S \bar{c}}{2 I_{y y}} \frac{180}{\pi} C_{m_{\delta_{e}}} \\
& g_{2,2}\left(\mathbf{x}_{2}\right)=\omega_{\delta_{e}}{ }^{2}
\end{aligned}
$$

$V_{c}[\mathrm{~m} / \mathrm{s}], \alpha[\mathrm{rad}], Q[\mathrm{rad} / \mathrm{s}], \Theta[\mathrm{rad}]$, and $\delta_{e}[\mathrm{rad}]$ are the velocity of center of gravity, angle of attack, pitch angular velocity, pitch angle, and elevator angle, respectively. $m[\mathrm{~kg}], S\left[\mathrm{~m}^{2}\right], \bar{c}[\mathrm{~m}]$, and $I_{y y}\left[\mathrm{~kg} \bullet \mathrm{m}^{2}\right]$ are the mass of the vehicle, wing area, mean aerodynamic chord, and moment of inertia around $\mathrm{Y}$ axis, respectively. $g\left[\mathrm{~m} / \mathrm{s}^{2}\right]$ and $\rho$ $\left[\mathrm{kg} / \mathrm{m}^{3}\right]$ are gravitational acceleration and air density, respectively. As for the aerodynamic coefficients, $C_{D}[-], C_{L}[-$ ], and $C_{m}[-]$ denote the coefficients of drag, lift, and pitching moment, respectively. $C_{m \delta e}\left[1 / \mathrm{deg}\right.$.], $C_{m q}$ [s/deg.], and $C_{m \dot{\alpha}}[\mathrm{s} / \mathrm{deg}$.] are the derivatives of pitching moment coefficient with respect to elevator angle, pitch angular velocity, and the angular velocity of angle of attack, respectively. As for the parameters of the elevator actuator, $\omega_{\delta e}[\mathrm{rad} / \mathrm{s}]$, $\zeta_{\delta e}[-]$ are natural frequency and damping coefficient of its dynamics, respectively. In this paper, $C_{L \delta e}$ and $C_{D \delta e}$ are assumed to be zero, and $C_{D}, C_{L}$, and $C_{m}$ are expressed as linear or quadratic functions of angle of attack.

\section{Design of Control Law Using Hierarchal Dynamic Inversion:-}

The transfer function of the actuator dynamics in the second hierarchy can be expressed as below.

$$
P_{\delta_{e}}=\frac{\delta_{e}}{\delta_{e_{\mathrm{com}}}}=\frac{\omega_{\delta_{e}}{ }^{2}}{s^{2}+2 \zeta_{\delta_{e}} \omega_{\delta_{e}} s+\omega_{\delta_{e}}{ }^{2}}
$$

The relative degree of the first hierarchy is two, and the second derivative of the angle of attack is written as eq. ( 47 ) using the relation in eq. ( 27 ).

$$
\begin{aligned}
\ddot{\alpha} & =L_{F_{1}}{ }^{2} h_{1}\left(\mathbf{x}_{1}\right)+\left[L_{G_{1}} L_{F_{1}} h_{1}\left(\mathbf{x}_{1}\right)\right] \delta_{e} \\
& =L_{F_{1}}{ }^{2} h_{1}\left(\mathbf{x}_{1}\right)+\left[L_{G_{1}} L_{F_{1}} h_{1}\left(\mathbf{x}_{1}\right)\right] \frac{\delta_{e}}{\delta_{e_{\text {com }}}} \delta_{e_{\text {com }}}
\end{aligned}
$$

where the command of the first hierarchy dynamics is given by eq. ( 48 ) based on eq. ( 21 ).

$$
\delta_{e_{\mathrm{com}}}=\left[L_{G_{1}} L_{F_{1}} h_{1}\left(\mathbf{x}_{1}\right)\right]^{-1}\left[\nu_{\alpha}-L^{2} F_{1} h_{1}\left(\mathbf{x}_{1}\right)\right]
$$


Equation ( 46 ) utilizing eq. ( 45 ) and eq. ( 48 ) gives eq. ( 49 ).

$$
\begin{aligned}
\ddot{\alpha} & =L_{F_{1}}{ }^{2} h_{1}\left(\mathbf{x}_{1}\right)+\left[L_{G_{1}} L_{F_{1}} h_{1}\left(\mathbf{x}_{1}\right)\right] P_{\delta_{e}}\left[L_{G_{1}} L_{F_{1}} h_{1}\left(\mathbf{x}_{1}\right)\right]^{-1}\left[v_{\alpha}-L_{F_{1}}{ }^{2} h_{1}\left(\mathbf{x}_{1}\right)\right] \\
& =L_{F_{1}}{ }^{2} h_{1}\left(\mathbf{x}_{1}\right)+P_{\delta_{e}}\left[v_{\alpha}-L_{F_{1}}{ }^{2} h_{1}\left(\mathbf{x}_{1}\right)\right] \\
& =L_{F_{1}}{ }^{2} h_{1}\left(\mathbf{x}_{1}\right)\left(1-P_{\delta_{e}}\right)+P_{\delta_{e}} v_{\alpha}
\end{aligned}
$$

The most right hand side of eq. ( 51 ) is divided into the nonlinear term and the linear term. If the effect of the nonlinear term is smaller than that of the linear term, the nonlinear term can be omitted in the following manner.

$$
\ddot{\alpha} \cong P_{\delta_{e}} v_{\alpha}
$$

where pseudo input is created by specifying the desired values of frequency $\omega_{\alpha}$ and damping coefficient $\zeta_{\alpha}$ as presented in eq. ( 53 ).

$$
v_{\alpha}=-\left(2 \zeta_{\alpha} \omega_{\alpha} \dot{\alpha}+\omega_{\alpha}^{2} \alpha\right)+\omega_{\alpha}{ }^{2} \alpha_{\text {com }}
$$

Subsequently, the Laplace transform of eq. ( 52 ) utilizing eq. ( 53 ) leads to eq. ( 54 ).

$$
\frac{\alpha}{\alpha_{\text {com }}}=\frac{\omega_{\alpha}{ }^{2} P_{\delta_{e}}}{s^{2}+2 \zeta_{\alpha} \omega_{\alpha} P_{\delta_{e}} s+\omega_{\alpha}{ }^{2} P_{\delta_{e}}}
$$

which gives LATF of the first hierarchy. This transfer function is constructed only by the actuator dynamics and the pseudo input, and the characteristics of LATF do not depend on the vehicle dynamics.

In addition, when the response speed of the output of the second hierarchy (elevator angle) is sufficiently higher than that of the first hierarchy (angle of attack), eq. ( 54 ) can be approximately reduced to the following equation.

$$
\frac{\alpha}{\alpha_{\text {com }}} \cong \frac{\omega_{\alpha}{ }^{2}}{s^{2}+2 \zeta_{\alpha} \omega_{\alpha} s+\omega_{\alpha}{ }^{2}}
$$

Transfer function of eq. ( 55 ) expresses ideal response of angle of attack, and it can be realized using the ideal eigenvalue specified by the user.

\section{Instantaneous Linearization of Hierarchical Dynamic Inversion:-}

In the previous section, the LATF was derived by ignoring the nonlinear term in eq. ( 49 ). In this section, in order to verify the accuracy of this approximation, a longitudinal dynamics model linearized around the equilibrium point (i.e., a trimmed state) is derived, and the result is compared with the LATF. Since the current values of the state is required for the hierarchical DI method to generate control command, the stability around the current state is evaluated via observing whether unstable eigenvalue exists or not. Each instantaneous state can be expressed by the summation of an equilibrium state and a small perturbation as follows.

$$
\begin{aligned}
& \mathbf{x}_{1}=\mathbf{x}_{1_{0}}+\Delta \mathbf{x}_{1} \\
& \mathbf{x}_{2}=\mathbf{x}_{2_{0}}+\Delta \mathbf{x}_{2} \\
& y_{1_{\text {com }}}=y_{1_{\text {com } 0}}+\Delta y_{1_{\text {com }}} \\
& y_{2_{\text {com }}}=y_{2_{\text {com } 0}}+\Delta y_{2_{\text {com }}}
\end{aligned}
$$

where each state vector and manipulated variable are

$$
\begin{aligned}
& \left\{\begin{aligned}
\mathbf{x}_{1} & =\left[\begin{array}{llll}
V_{c} & \alpha & Q & \Theta
\end{array}\right]^{T} \\
\mathbf{x}_{1_{0}} & =\left[\begin{array}{llll}
V_{c_{0}} & \alpha_{0} & Q_{0} & \Theta_{0}
\end{array}\right]^{T} \\
\Delta \mathbf{x}_{1} & =\left[\begin{array}{llll}
v_{c} & \alpha_{b} & q & \theta
\end{array}\right]^{T}
\end{aligned}\right. \\
& \left\{\begin{array}{l}
\mathbf{x}_{2}=\left[\begin{array}{ll}
\delta_{e} & \dot{\delta}_{e}
\end{array}\right]^{T} \\
\mathbf{x}_{2_{0}}=\left[\begin{array}{ll}
\delta_{e_{0}} & 0
\end{array}\right]^{T} \\
\Delta \mathbf{x}_{2}=\left[\begin{array}{ll}
\delta_{e_{b}} & \dot{\delta}_{e_{b}}
\end{array}\right]^{T}
\end{array}\right. \\
& \begin{cases}y_{1} & =\alpha \\
y_{2} & =\delta_{e}\end{cases}
\end{aligned}
$$

Linearized equation for generating the command of control input is obtained from eq. ( 48 ) as follows. 


$$
\begin{aligned}
& y_{2_{\text {com }}}=\frac{1}{L_{G_{1}} L_{F_{1}} h_{1}\left(\mathbf{x}_{1}\right)}\left[v_{\alpha}\left(\mathbf{x}_{1}, y_{1_{\mathrm{com}}}\right)-L_{F_{1}}^{2} h_{1}\left(\mathbf{x}_{1}\right)\right] \\
& =\frac{1}{L_{G_{1}} L_{F_{1}} h_{1}\left(\mathbf{x}_{1_{0}}+\Delta \mathbf{x}_{1}\right)}\left[\nu_{\alpha}\left(\mathbf{x}_{1_{0}}+\Delta \mathbf{x}_{1}, y_{1_{\mathrm{com} n}}+\Delta y_{\mathrm{c}_{\mathrm{com}}}\right)-L_{F_{1}}{ }^{2} h_{1}\left(\mathbf{x}_{1_{0}}+\Delta \mathbf{x}_{1}\right)\right] \\
& y_{2_{\text {com } 0}}+\Delta y_{2_{\text {com }}}=\frac{1}{L_{G_{1}} L_{F_{1}} h_{1}\left(\mathbf{x}_{1_{0}}\right)}\left[v_{\alpha}\left(\mathbf{x}_{1_{0}}, y_{1_{\text {con } 0}}\right)-L_{F_{1}}{ }^{2} h_{1}\left(\mathbf{x}_{1_{0}}\right)\right] \\
& +\left(L_{G_{1}} L_{F_{1}} h_{1}\left(\mathbf{x}_{1_{0}}\right)\right)^{-2}\left[\left(L_{G_{1}} L_{F_{1}} h_{1}\left(\mathbf{x}_{1_{0}}\right)\right)\left(\frac{\partial v_{\alpha}\left(\mathbf{x}_{1_{0}}, y_{1_{\text {como }}}\right)}{\partial \mathbf{x}_{1}}-\frac{\partial L_{F_{1}}{ }^{2} h_{1}\left(\mathbf{x}_{1_{0}}\right)}{\partial \mathbf{x}_{1}}\right)\right. \\
& \left.-\frac{\partial L_{G_{1}} L_{F_{1}} h_{1}\left(\mathbf{x}_{1_{0}}\right)}{\partial \mathbf{x}_{1}}\left(v_{\alpha}\left(\mathbf{x}_{1_{0}}, y_{1_{\text {como }}}\right)-L_{F_{1}}{ }^{2} h_{1}\left(\mathbf{x}_{1_{0}}\right)\right)\right] \Delta \mathbf{x}_{1} \\
& +\left(L_{G_{1}} L_{F_{1}} h_{1}\left(\mathbf{x}_{1_{0}}\right)\right)^{-1} \frac{\partial v_{\alpha}\left(\mathbf{x}_{1_{0}}, y_{1_{\text {como }}}\right)}{\partial y_{1_{\text {com }}}} \Delta y_{1_{\text {com }}}+O\left(\Delta \mathbf{x}_{1}^{2}\right)+O\left(\Delta y_{1_{\text {com }}}{ }^{2}\right)
\end{aligned}
$$

Equation ( 62 ) is a Taylor's expansion of eq. ( 60 ). Here, when $y_{2_{\mathrm{com} 0}}$ is equipment state, the following equation will be established.

$$
y_{2_{\text {como }}}=\frac{1}{L_{G_{1}} L_{F_{1}} h_{1}\left(\mathbf{x}_{1_{0}}\right)}\left[v_{\alpha}\left(\mathbf{x}_{1_{0}}, y_{1_{\text {como } 0}}\right)-L_{F_{1}}{ }^{2} h_{1}\left(\mathbf{x}_{1_{0}}\right)\right]
$$

In addition, if eq. ( 62 ) is able to approximate linearization, second order terms of the $\Delta \mathbf{x}_{1}$ and $\Delta y_{1_{\text {com }}}$ can be ignored. Small perturbation of $\Delta y_{2_{\text {com }}}$ is able to transfer to the eq. ( 65 ).

$$
\begin{aligned}
\Delta y_{2_{\text {com }}}= & \left(L_{G_{1}} L_{F_{1}} h_{1}\left(\mathbf{x}_{1_{0}}\right)\right)^{-2}\left[\left(L_{G_{1}} L_{F_{1}} h_{1}\left(\mathbf{x}_{1_{0}}\right)\right)\left(\frac{\partial v_{\alpha}\left(\mathbf{x}_{1_{1}}, y_{1_{\mathrm{com}}}\right)}{\partial \mathbf{x}_{1}}-\frac{\partial L_{F_{1}}{ }^{2} h_{1}\left(\mathbf{x}_{1_{0}}\right)}{\partial \mathbf{x}_{1}}\right)\right. \\
& \left.-\frac{\partial L_{G_{1}} L_{F_{1}} h_{1}\left(\mathbf{x}_{1_{0}}\right)}{\partial \mathbf{x}_{1}}\left(v_{\alpha}\left(\mathbf{x}_{1_{0}}, y_{1_{\mathrm{com}}}\right)-L_{F_{1}}{ }^{2} h_{1}\left(\mathbf{x}_{1_{0}}\right)\right)\right] \Delta \mathbf{x}_{1} \\
& +\left(L_{G_{1}} L_{F_{1}} h_{1}\left(\mathbf{x}_{1_{0}}\right)\right)^{-1} \frac{\partial v_{\alpha}\left(\mathbf{x}_{1_{0}}, y_{1_{\mathrm{com}}}\right)}{\partial y_{1_{\mathrm{com}}}} \Delta y_{1_{\mathrm{com}}} \\
= & \left(L_{G_{1}} L_{F_{1}} h_{1}\left(\mathbf{x}_{1}\right)\right)^{-1}\left[\frac{\partial v_{\alpha}\left(\mathbf{x}_{1_{0}}, y_{1_{\mathrm{com}}}\right)}{\partial \mathbf{x}_{1}}-\frac{\partial L_{F_{1}}{ }^{2} h_{1}\left(\mathbf{x}_{1}\right)}{\partial \mathbf{x}_{1}}-y_{2_{\mathrm{com} 0}} \frac{\partial L_{G_{1}} L_{F_{1}} h_{1}\left(\mathbf{x}_{1}\right)}{\partial \mathbf{x}_{1}}\right] \Delta \mathbf{x}_{1} \\
& +\left(L_{G_{1}} L_{F_{1}} h_{1}\left(\mathbf{x}_{1}\right)\right)^{-1} \frac{\partial v_{\alpha}\left(\mathbf{x}_{1_{0}}, y_{1_{\mathrm{com}}}\right)}{\partial y_{1_{\mathrm{com}}}} \Delta y_{1_{\mathrm{com}}}
\end{aligned}
$$

Therefore, equilibrium of elevator command and small perturbation of one are the as following eq. ( 66 ), and eq. ( 67 ) by replacing $y_{2_{\text {com }}}$ with $\delta_{e_{\text {com }}}$. And equilibrium of angle of attack command and small perturbation of one are replacing $y_{1_{\text {com }}}$ with $\alpha_{\text {com }}$.

$$
\begin{aligned}
\delta_{e_{\text {como }}}= & \frac{1}{L_{G_{1}} L_{F_{1}} h_{1}\left(\mathbf{x}_{1_{0}}\right)}\left[v_{\alpha}\left(\mathbf{x}_{1_{0}}, \alpha_{\text {como }}\right)-L_{F_{1}}{ }^{2} h_{1}\left(\mathbf{x}_{1_{0}}\right)\right] \\
= & \left(L_{G_{1}} L_{F_{1}} h_{1}\left(\mathbf{x}_{1}\right)\right)^{-1}\left[\frac{\partial v_{\alpha}\left(\mathbf{x}_{1_{0}}, \alpha_{\text {com }}\right)}{\partial \mathbf{x}_{1}}-\frac{\partial L_{F_{1}}{ }^{2} h_{1}\left(\mathbf{x}_{1}\right)}{\partial \mathbf{x}_{1}}-\delta_{e_{\text {como }}} \frac{\partial L_{G_{1}} L_{F_{1}} h_{1}\left(\mathbf{x}_{1}\right)}{\partial \mathbf{x}_{1}}\right] \Delta \mathbf{x}_{1} \\
& +\left(L_{G_{1}} L_{F_{1}} h_{1}\left(\mathbf{x}_{1}\right)\right)^{-1} \frac{\partial v_{\alpha}\left(\mathbf{x}_{1_{0}}, \alpha\right. \text { com }}{\partial \alpha_{\text {com }}} \Delta \alpha_{\text {com }}
\end{aligned}
$$

The transfer function of the second hierarchy is expressed as follows.

$$
P_{\delta_{e}}=\frac{\delta_{e}}{\delta_{e_{\mathrm{com}}}}=\frac{{\omega_{\delta_{e}}}^{2}}{s^{2}+2 \zeta_{\delta_{e}} \omega_{\delta_{e}} s+\omega_{\delta_{e}}{ }^{2}}
$$

Using eqs. ( 46 ), ( 66 ), ( 67 ), and ( 68 ), linearized dynamics for angle of attack is obtained as follows.

$$
\begin{aligned}
\ddot{\alpha} & =L^{2} F_{1} h_{1}\left(\mathbf{x}_{1}\right)+L_{G_{1}} L_{F_{1}} h_{1}\left(\mathbf{x}_{1}\right) \delta_{e} \\
& =L^{2} F_{1} h_{1}\left(\mathbf{x}_{1}\right)+L_{G_{1}} L_{F_{1}} h_{1}\left(\mathbf{x}_{1}\right) P_{\delta_{e}} \delta_{e_{\mathrm{com}}} \\
& =L^{2} F_{1} h_{1}\left(\mathbf{x}_{1_{0}}+\Delta \mathbf{x}_{1}\right)+L_{G_{1}} L_{F_{1}} h_{1}\left(\mathbf{x}_{1_{0}}+\Delta \mathbf{x}_{1}\right) P_{\delta_{e}}\left(\delta_{e_{\mathrm{com}}}+\Delta \delta_{e}\right)
\end{aligned}
$$


Taylor's expansion is performed for eq. ( 71 ), and eqs. ( 72 ) and ( 73 ) are derived with the assumptions of equilibrium states of $\ddot{\alpha}$ and ignorance of perturbation larger than the second order.

$$
\begin{aligned}
& 0=L_{F_{1}}^{2} h_{1}\left(\mathbf{x}_{1_{0}}\right)+L_{G_{1}} L_{F_{1}} h_{1}\left(\mathbf{x}_{1_{0}}\right) P_{\delta_{e}} \delta_{e_{\text {com }}} \\
& \ddot{\alpha}_{b}=\left[\frac{\partial L_{F_{1}}^{2} h_{1}\left(\mathbf{x}_{1_{0}}\right)}{\partial \mathbf{x}_{1}}+\frac{\partial L_{G_{1}} L_{F_{1}} h_{1}\left(\mathbf{x}_{1_{0}}\right)}{\partial \mathbf{x}_{1}} P_{\delta_{e}} \delta_{e_{\text {como }}}\right] \Delta \mathbf{x}_{1}+L_{G_{1}} L_{F_{1}} h_{1}\left(\mathbf{x}_{1_{0}}\right) P_{\delta_{e}} \Delta \delta_{e_{\text {com }}}
\end{aligned}
$$

Substituting eq. ( 73 ) into eq. ( 67 ) results in eq. ( 74 ).

$$
\ddot{\alpha}_{b}=\left[\frac{\partial L_{F_{1}}^{2} h_{1}(\alpha)}{\partial \mathbf{x}_{1}}\left(1-P_{\delta_{e}}\right)+P_{\delta_{e}} \frac{\partial v_{\alpha}\left(\mathbf{x}_{1_{0}}, \alpha\right. \text { com }}{\partial \mathbf{x}_{1}}\right] \Delta \mathbf{x}_{1}+P_{\delta_{e}} \frac{\partial v_{\alpha}\left(\mathbf{x}_{1_{0}}, \alpha_{\mathrm{com}}\right)}{\partial \alpha_{\text {com }}} \Delta \alpha_{\text {com }}
$$

By using the pseudo input defined in eq. ( 53 ), eq. ( 74 ) is rewritten as follows.

$$
\begin{aligned}
\ddot{\alpha}_{b} & =\left[\frac{\partial L_{F_{1}}{ }^{2} h_{1}(\alpha)}{\partial \mathbf{x}_{1}}\left(1-P_{\delta_{e}}\right)-P_{\delta_{e}}\left(\omega_{\alpha}{ }^{2} \frac{\partial \alpha}{\partial \mathbf{x}_{1}}+2 \zeta_{\alpha} \omega_{\alpha} \frac{\partial \dot{\alpha}}{\partial \mathbf{x}_{1}}\right)\right] \Delta \mathbf{x}_{1}+P_{\delta_{e}} \omega_{\alpha}{ }^{2} \Delta \alpha_{\mathrm{com}} \\
= & {\left[\begin{array}{l}
\frac{\partial L_{F_{1}}{ }^{2} h_{1}(\alpha)}{\partial V_{c}}\left(1-P_{\delta_{e}}\right)-2 \zeta_{\alpha} \omega_{\alpha} \frac{\partial \dot{\alpha}}{\partial V_{c}} P_{\delta_{e}} \\
\frac{\partial L_{F_{1}}{ }^{2} h_{1}(\alpha)}{\partial \alpha}\left(1-P_{\delta_{e}}\right)-\left(2 \zeta_{\alpha} \omega_{\alpha} \frac{\partial \dot{\alpha}}{\partial \alpha}+\omega_{\alpha}{ }^{2}\right) P_{\delta_{e}} \\
\frac{\partial L_{F_{1}}{ }^{2} h_{1}(\alpha)}{\partial Q}\left(1-P_{\delta_{e}}\right)-2 \zeta_{\alpha} \omega_{\alpha} \frac{\partial \dot{\alpha}}{\partial Q} P_{\delta_{e}} \\
\frac{\partial L_{F_{1}}{ }^{2} h_{1}(\alpha)}{\partial \Theta}\left(1-P_{\delta_{e}}\right)-2 \zeta_{\alpha} \omega_{\alpha} \frac{\partial \dot{\alpha}}{\partial \Theta} P_{\delta_{e}}
\end{array}\right]^{T}\left[\begin{array}{c}
v_{c} \\
\alpha_{b} \\
q \\
\theta
\end{array}\right]+P_{\delta_{e}} \omega_{\alpha}{ }^{2} \Delta \alpha_{\mathrm{com}} }
\end{aligned}
$$

As for the other states,

$$
\begin{aligned}
& \dot{v}_{c}=\frac{\partial}{\partial \mathbf{x}_{1}} f_{1,1} \Delta \mathbf{x}_{1}=\left[\begin{array}{l}
\frac{\partial f_{1,1}}{\partial V_{c}} \\
\frac{\partial f_{1,1}}{\partial \alpha} \\
\frac{\partial f_{1,1}}{\partial Q} \\
\frac{\partial f_{1,1}}{\partial \Theta}
\end{array}\right]^{T}\left[\begin{array}{c}
v_{c} \\
\alpha_{b} \\
q \\
\theta
\end{array}\right] \\
& \dot{\alpha}_{b}=\frac{\partial}{\partial \mathbf{x}_{1}} f_{1,2} \Delta \mathbf{x}_{1}=\left[\begin{array}{c}
\frac{\partial f_{1,2}}{\partial V_{c}} \\
\frac{\partial f_{1,2}}{\partial \alpha} \\
\frac{\partial f_{1,2}}{\partial Q} \\
\frac{\partial f_{1,2}}{\partial \Theta}
\end{array}\right]^{T}\left[\begin{array}{c}
v_{c} \\
\alpha_{b} \\
q \\
\theta
\end{array}\right] \\
& \dot{\theta}=\frac{\partial}{\partial \mathbf{x}_{1}} f_{1,4} \Delta \mathbf{x}_{1}=\left[\begin{array}{c}
\frac{\partial f_{1,4}}{\partial V_{c}} \\
\frac{\partial f_{1,4}}{\partial \alpha} \\
\frac{\partial f_{1,4}}{\partial Q} \\
\dot{Q}=f_{1,3}\left(\mathbf{x}_{1}\right)+g_{1,3}\left(\mathbf{x}_{1}\right) \delta_{e} \\
=f_{1,3}\left(\mathbf{x}_{1}\right)+g_{1,3}\left(\mathbf{x}_{1}\right) P_{\delta_{e}} \delta_{e_{\mathrm{com}}} \\
{[\Theta}
\end{array}\right]^{T}\left[\begin{array}{c}
v_{c} \\
\alpha_{b} \\
q \\
\theta
\end{array}\right]
\end{aligned}
$$




$$
\begin{aligned}
& \dot{q}=\left[\frac{\partial f_{1,3}}{\partial \mathbf{x}_{1}}\left(\mathbf{x}_{1_{0}}\right)+\frac{\partial g_{1,3}}{\partial \mathbf{x}_{1}}\left(\mathbf{x}_{1_{0}}\right) P_{\delta_{e}} \delta_{e_{\text {como }}}\left(\mathbf{x}_{1_{0}}, \alpha_{\text {cono }}\right)\right] \Delta \mathbf{x}_{1}+g_{1,3}\left(\mathbf{x}_{1_{0}}\right) P_{\delta_{e}} \Delta \delta_{e_{\text {com }}}
\end{aligned}
$$

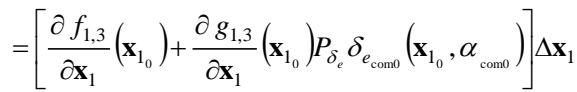

$$
\begin{aligned}
& +g_{1,3}\left(\mathbf{x}_{1_{0}}\right) P_{\delta_{e}}\left(L_{G_{1}} L_{F_{1}} h_{1}\left(\mathbf{x}_{1_{0}}\right)\right)^{-1}\left[\frac{\partial v_{\alpha}}{\partial \mathbf{x}_{1}}\left(\mathbf{x}_{1_{0}}, \alpha_{\text {conon }}\right)-\frac{\partial}{\partial \mathbf{x}_{1}}\left(L_{F_{1}}{ }^{2} h_{1}\left(\mathbf{x}_{1_{0}}\right)\right)-\delta_{e_{\text {cono }}} \frac{\partial}{\partial \mathbf{x}_{1}}\left(L_{G_{1}} L_{F_{1}} h_{1}\left(\mathbf{x}_{1_{0}}\right)\right)\right] \Delta \mathbf{x}_{1} \\
& +g_{1,3}\left(\mathbf{x}_{1_{0}}\right) P_{\delta_{e}}\left(L_{G_{1}} L_{F_{1}} h_{1}\left(\mathbf{x}_{1_{0}}\right)\right)^{-1} \frac{\partial v_{\alpha}}{\partial \alpha_{\text {com }}}\left(\mathbf{x}_{1_{0}}, \alpha_{\text {como }}\right) \Delta \alpha_{\text {com }} \\
& =\left[\frac{\partial f_{1,3}\left(\mathbf{x}_{1_{0}}\right)}{\partial \mathbf{x}_{1}}+P_{\delta_{e}} \delta_{e_{\text {como }}} \frac{\partial g_{1,3}\left(\mathbf{x}_{1_{0}}\right)}{\partial \mathbf{x}_{1}}+P_{\delta_{e}} \frac{g_{1,3} \mathbf{x}_{1_{0}}}{L_{G_{1}} L_{F_{1}} h_{1}\left(\mathbf{x}_{1}\right)} \frac{\partial v_{\alpha}\left(\mathbf{x}_{1_{0}}, \alpha_{\text {com }}\right)}{\partial \mathbf{x}_{1}}\right. \\
& \left.-\frac{g_{1,3}\left(\mathbf{x}_{1_{0}}\right)}{L_{G_{1}} L_{F_{1}} h_{1}\left(\mathbf{x}_{1_{0}}\right)} \frac{\partial}{\partial \mathbf{x}_{1}}\left(L_{F_{1}}{ }^{2} h_{1}\left(\mathbf{x}_{1_{0}}\right)\right)-\frac{g_{1,3} \mathbf{x}_{1_{0}}}{L_{G_{1}} L_{F_{1}} h_{1}\left(\mathbf{x}_{1_{0}}\right)} P_{\delta_{e}} \delta_{e_{\text {cono }}} \frac{\partial}{\partial \mathbf{x}_{1}}\left(L_{G_{1}} L_{F_{1}} h_{1}\left(\mathbf{x}_{1_{0}}\right)\right)\right] \Delta \mathbf{x}_{1} \\
& +P_{\delta_{e}} \frac{g_{1,3}\left(\mathbf{x}_{1_{0}}\right)}{L_{G_{1}} L_{F_{1}} h_{1}\left(\mathbf{x}_{1_{0}}\right)} \frac{\partial v_{\alpha}\left(\mathbf{x}_{1_{0}}, \alpha_{\text {com }}\right)}{\partial \alpha_{\text {com }}} \Delta \alpha_{\text {com }} \\
& =\left\{\left[\frac{\partial f_{1,3}\left(\mathbf{x}_{1_{0}}\right)}{\partial \mathbf{x}_{1}}-\frac{g_{1,3}\left(\mathbf{x}_{1_{1}}\right)}{L_{G_{1}} L_{F_{1}} h_{1}\left(\mathbf{x}_{1_{0}}\right)}\right) \frac{\partial}{\partial \mathbf{x}_{1}}\left(L_{F_{1}}{ }^{2} h_{1}\left(\mathbf{x}_{1_{0}}\right)\right)\right] \\
& +\left[\frac{\partial g_{1,3}\left(\mathbf{x}_{1_{0}}\right)}{\partial \mathbf{x}_{1}}-\frac{g_{1,3}\left(\mathbf{x}_{1_{0}}\right)}{L_{G_{1}} L_{F_{1}} h_{1}\left(\mathbf{x}_{1_{0}}\right)} \frac{\partial}{\partial \mathbf{x}_{1}}\left(L_{G_{1}} L_{F_{1}} h_{1}\left(\mathbf{x}_{1_{0}}\right)\right)\right] P_{\delta_{e}} \frac{v_{\alpha}\left(\mathbf{x}_{1_{0}}, \alpha_{\text {cono }}\right)-L_{F_{1}}{ }^{2} h_{1}\left(\mathbf{x}_{1_{0}}\right)}{L_{G_{1}} L_{F_{1}} h_{1}\left(\mathbf{x}_{1_{0}}\right)} \\
& \left.+P_{\delta_{e}} \frac{g_{1,3}\left(\mathbf{x}_{1_{0}}\right)}{L_{G_{1}} L_{F_{1}} h_{1}\left(\mathbf{x}_{1_{0}}\right)} \frac{\partial v_{\alpha}\left(\mathbf{x}_{1_{0}}, \alpha_{\text {como }}\right)}{\partial \mathbf{x}_{1}}\right\} \Delta \mathbf{x}_{1} \\
& +P_{\delta_{e}} \frac{g_{1,3}\left(\mathbf{x}_{1_{0}}\right)}{L_{G_{1}} L_{F_{1}} h_{1}\left(\mathbf{x}_{1_{0}}\right)} \frac{\partial v_{\alpha}\left(\mathbf{x}_{1_{0}}, \alpha_{\mathrm{com} 0}\right)}{\partial \alpha_{\mathrm{com}}} \Delta \alpha_{\mathrm{com}}
\end{aligned}
$$

Whereas the derivative of pitch angular velocity is complex due to the influence by the second hierarchy, some terms in eq. ( 85 ) can be simplified using the following relations:

$$
\begin{aligned}
& L_{G_{1}} L_{F_{1}} h_{1}\left(\mathbf{x}_{1_{0}}\right)=J_{23} g_{1,3}\left(\mathbf{x}_{1_{0}}\right) \\
& L_{F_{1}}{ }^{2} h_{1}\left(\mathbf{x}_{1_{0}}\right)=J_{21} f_{1,1}+J_{22} f_{1,2}+J_{23} f_{1,3}+J_{24} f_{2,4}
\end{aligned}
$$

where $J_{i j}$ is the $(i, j)$ th component of the Jacobian matrix of $F_{1}\left(\mathbf{x}_{1}\right)$ shown below.

$$
\begin{aligned}
J & =\left[\left.\begin{array}{llll}
\frac{\partial f_{1,1}\left(\mathbf{x}_{1}\right)}{\partial V_{\varepsilon}} & \frac{\partial f_{1,1}\left(\mathbf{x}_{1}\right)}{\partial \alpha} & \frac{\partial f_{1,1}\left(\mathbf{x}_{1}\right)}{\partial Q} & \frac{\partial f_{1,1}\left(\mathbf{x}_{1}\right)}{\partial \Theta} \\
\frac{\partial f_{1,2}\left(\mathbf{x}_{1}\right)}{\partial V_{(}} & \frac{\partial f_{1,2}\left(\mathbf{x}_{1}\right)}{\partial \alpha} & \frac{\partial f_{1,2}\left(\mathbf{x}_{1}\right)}{\partial Q} & \frac{\partial f_{1,2}\left(\mathbf{x}_{1}\right)}{\partial \Theta} \\
\frac{\partial f_{1,3}\left(\mathbf{x}_{1}\right)}{\partial V_{E}} & \frac{\partial f_{1,3}\left(\mathbf{x}_{1}\right)}{\partial \alpha} & \frac{\partial f_{1,3}\left(\mathbf{x}_{1}\right)}{\partial Q} & \frac{\partial f_{1,3}\left(\mathbf{x}_{1}\right)}{\partial \Theta} \\
\frac{\partial f_{1,4}\left(\mathbf{x}_{1}\right)}{\partial V_{c}} & \frac{\partial f_{1,4}\left(\mathbf{x}_{1}\right)}{\partial \alpha} & \frac{\partial f_{1,4}\left(\mathbf{x}_{1}\right)}{\partial Q} & \frac{\partial f_{1,4}\left(\mathbf{x}_{1}\right)}{\partial \Theta}
\end{array}\right|_{\mathbf{x}_{1}=\mathbf{x}_{10}}\right. \\
= & {\left[\left.\begin{array}{llll}
J_{11} & J_{12} & J_{13} & J_{14} \\
J_{21} & J_{22} & J_{23} & J_{24} \\
J_{31} & J_{32} & J_{33} & J_{34} \\
J_{41} & J_{42} & J_{43} & J_{44}
\end{array}\right|_{\mathbf{x}_{1}=\mathbf{x}_{10}}\right.} \\
J_{11} & =-\frac{\rho V_{c_{0}} S}{m} C_{D}-\left.\frac{\rho V_{c_{0}}{ }^{2} S}{2 m} \frac{M_{0}}{V_{c_{0}}} \frac{\partial C_{D}}{\partial M}\right|_{M=M_{0}} \\
J_{12} & =g \cos \left(\Theta_{0}-\alpha_{0}\right)-\left.\frac{\rho V_{c_{0}}{ }^{2} S}{2 m} \frac{\partial C_{D}}{\partial \alpha}\right|_{\alpha=\alpha_{0}} \\
J_{13} & =0 \\
J_{14} & =-g \cos \left(\Theta_{0}-\alpha_{0}\right)
\end{aligned}
$$




$$
\begin{aligned}
J_{21}= & -\frac{g}{V_{c_{0}}{ }^{2}} \cos \left(\Theta_{0}-\alpha_{0}\right)-\frac{\rho S}{2 m} C_{L}-\left.\frac{\rho V_{c_{0}} S}{2 m} \frac{M_{0}}{V_{c_{0}}} \frac{\partial C_{L}}{\partial M}\right|_{M=M_{0}} \\
J_{22}= & \frac{g}{V_{c_{0}}} \sin \left(\Theta_{0}-\alpha_{0}\right)-\left.\frac{\rho V_{c_{0}} S}{2 m} \frac{\partial C_{L}}{\partial \alpha}\right|_{\alpha=\alpha_{0}} \\
J_{23}= & 1 \\
J_{24}= & -\frac{g}{V_{c_{0}}} \sin \left(\Theta_{0}-\alpha_{0}\right) \\
J_{31}= & \frac{\rho V_{c_{0}} S \bar{c}}{I_{y y}}\left[C_{m}+\frac{\bar{c}}{2 V_{c_{0}}}\left(C_{m_{q}} Q_{0}+C_{m_{\dot{\alpha}}} \dot{\alpha}\right)\right] \\
& +\frac{\rho V_{c_{0}}{ }^{2} S \bar{c}}{2 I_{y y}}\left[\left.\frac{M_{0}}{V_{c_{0}}} \frac{\partial C_{m}}{\partial M}\right|_{M=M_{0}}-\frac{\bar{c}}{2 V_{c_{0}}{ }^{2}}\left(C_{m_{q}} Q_{0}+C_{m_{\dot{\alpha}}} \dot{\alpha}\right)+\left.\frac{\bar{c}}{2 V_{c_{0}}} C_{m_{\dot{\alpha}}} \frac{\partial \dot{\alpha}}{\partial V_{c}}\right|_{V_{c}=V_{c_{0}}}\right] \\
J_{32}= & \frac{\rho V_{c_{0}}{ }^{2} S \bar{c}}{2 I_{y y}}\left(\left.\frac{\partial C_{m}}{\partial \alpha}\right|_{\alpha=\alpha_{0}}+\left.\frac{\bar{c}}{2 V_{c_{0}}} C_{m_{\dot{\alpha}}} \frac{\partial \dot{\alpha}}{\partial \alpha}\right|_{\alpha=\alpha_{0}}\right) \\
J_{33}= & \frac{\rho V_{c_{0}} S \bar{c}^{2}}{4 I_{y y}}\left(C_{m_{q}}+\left.C_{m_{\dot{\alpha}}} \frac{\partial \dot{\alpha}}{\partial Q}\right|_{Q=Q_{0}}\right) \\
J_{34}= & -\left.\frac{\rho V_{c_{0}} S \bar{c}^{2}}{4 I_{y y}} C_{m_{\dot{\alpha}}} \frac{\partial \dot{\alpha}}{\partial \Theta}\right|_{\Theta=\Theta_{0}} \\
J_{41}= & 0 \\
J_{42}= & 0 \\
J_{43}= & 1 \\
J_{44}= & 0 \\
C_{y-2} &
\end{aligned}
$$

Then, eq. ( 85 ) can be reduced to

$$
\begin{aligned}
\dot{q}= & \left\{\left[\frac{\partial f_{1,3}}{\partial \mathbf{x}_{1}}-\frac{g_{1,3}}{L_{G_{1}} L_{F_{1}} h_{1}\left(\mathbf{x}_{1}\right)} \frac{\partial}{\partial \mathbf{x}_{1}}\left(L_{F_{1}}{ }^{2} h_{1}\left(\mathbf{x}_{1}\right)\right)\right]\right. \\
& \left.+\left[\frac{\partial g_{1,3}}{\partial \mathbf{x}_{1}}-\frac{g_{1,3}}{L_{G_{1}} L_{F_{1}} h_{1}\left(\mathbf{x}_{1}\right)} \frac{\partial}{\partial \mathbf{x}_{1}}\left(L_{G_{1}} L_{F_{1}} h_{1}\left(\mathbf{x}_{1}\right)\right)\right] \frac{v_{\alpha}-L_{F_{1}}{ }^{2} h_{1}\left(\mathbf{x}_{1}\right)}{L_{G_{1}} L_{F_{1}} h_{1}\left(\mathbf{x}_{1}\right)} P_{\delta_{e}}+P_{\delta_{e}} \frac{g_{1,3}}{L_{G_{1}} L_{F_{1}} h_{1}\left(\mathbf{x}_{1}\right)} \frac{\partial v_{\alpha}}{\partial \mathbf{x}_{1}}\right\} \Delta \mathbf{x}_{1} \\
& +P_{\delta_{e}} \frac{g_{1,3}}{L_{G_{1}} L_{F_{1}} h_{1}\left(\mathbf{x}_{1}\right)} \frac{\partial v_{\alpha}}{\partial \alpha_{\mathrm{com}}} \Delta \alpha_{\mathrm{com}} \\
= & \left\{\left[\frac{\partial f_{1,3}}{\partial \mathbf{x}_{1}}-\frac{g_{1,3}}{J_{23} g_{1,3}} \frac{\partial}{\partial \mathbf{x}_{1}}\left(\sum_{i=1}^{4} J_{2 i} f_{1, i}\right) P_{\delta_{e}}\right]\right. \\
& \left.+\frac{1}{J_{23} g_{1,3}}\left[\frac{\partial g_{1,3}}{\partial \mathbf{x}_{1}}-\frac{g_{1,3}}{J_{23} g_{1,3}} \frac{\partial\left(J_{23} g_{1,3}\right)}{\partial \mathbf{x}_{1}}\right]\left[v_{\alpha}-\left(\sum_{i=1}^{4} J_{2 i} f_{1, i}\right)\right] P_{\delta_{e}}+P_{\delta_{e}} \frac{\partial v_{\alpha}}{\partial \mathbf{x}_{1}}\right\} \Delta \mathbf{x}_{1} \\
& +P_{\delta_{e}} \frac{g_{1,3}}{J_{23} g_{1,3}} \frac{\partial v_{\alpha}}{\partial \alpha_{\mathrm{com}}} \Delta \alpha_{\mathrm{com}}
\end{aligned}
$$

Since $J_{23}=1$, eq. ( 85 ) is further simplified to

$$
\begin{aligned}
\dot{q}= & \left\{\left[\frac{\partial f_{1,3}}{\partial \mathbf{x}_{1}}-\frac{\partial}{\partial \mathbf{x}_{1}}\left(\sum_{i=1}^{4} J_{2 i} f_{1, i}\right) P_{\delta_{e}}\right]\right. \\
& \left.+\frac{1}{g_{1,3}}\left[\frac{\partial g_{1,3}}{\partial \mathbf{x}_{1}}-\frac{\partial g_{1,3}}{\partial \mathbf{x}_{1}}\right]\left[v_{\alpha}-\left(\sum_{i=1}^{4} J_{2 i} f_{1, i}\right)\right] P_{\delta_{e}}+P_{\delta_{e}} \frac{\partial v_{\alpha}}{\partial \mathbf{x}_{1}}\right\} \Delta \mathbf{x}_{1} \\
& +P_{\delta_{e}} \frac{\partial v_{\alpha}}{\partial \alpha_{\mathrm{com}}} \Delta \alpha_{\mathrm{com}} \\
= & \left\{\left[\frac{\partial f_{1,3}}{\partial \mathbf{x}_{1}}-\frac{\partial}{\partial \mathbf{x}_{1}}\left(\sum_{i=1}^{4} J_{2 i} f_{1, i}\right) P_{\delta_{e}}\right]+P_{\delta_{e}} \frac{\partial v_{\alpha}}{\partial \mathbf{x}_{1}}\right\} \Delta \mathbf{x}_{1}+P_{\delta_{e}} \frac{\partial v_{\alpha}}{\partial \alpha_{\mathrm{com}}} \Delta \alpha_{\mathrm{com}}
\end{aligned}
$$




$$
=\left[\begin{array}{l}
J_{31}-\left(J_{21}{ }^{\prime}+2 \zeta_{\alpha} \omega_{\alpha} J_{21}\right) P_{\delta_{e}} \\
J_{32}-\left(J_{22}{ }^{\prime}+2 \zeta_{\alpha} \omega_{\alpha} J_{22}+\omega_{\alpha}{ }^{2}\right) P_{\delta_{e}} \\
J_{33}-\left(J_{23}{ }^{\prime}+2 \zeta_{\alpha} \omega_{\alpha} J_{23}\right) P_{\delta_{e}} \\
J_{34}-\left(J_{24}{ }^{\prime}+2 \zeta_{\alpha} \omega_{\alpha} J_{24}\right) P_{\delta_{e}}
\end{array}\right]^{T}\left[\begin{array}{c}
v_{c} \\
\alpha_{b} \\
q \\
\theta
\end{array}\right]+P_{\delta_{e}} \omega_{\alpha}{ }^{2} \Delta \alpha_{\mathrm{com}}
$$

where

$$
\begin{aligned}
& J_{21}{ }^{\prime}=\frac{\partial}{\partial V_{c}}\left(J_{21} f_{1,1}+J_{22} f_{1,2}+J_{23} f_{1,3}+J_{24} f_{1,4}\right) \\
& J_{22}{ }^{\prime}=\frac{\partial}{\partial \alpha}\left(J_{21} f_{1,1}+J_{22} f_{1,2}+J_{23} f_{1,3}+J_{24} f_{1,4}\right) \\
& J_{23}{ }^{\prime}=\frac{\partial}{\partial Q}\left(J_{21} f_{1,1}+J_{22} f_{1,2}+J_{23} f_{1,3}+J_{24} f_{1,4}\right) \\
& J_{24}{ }^{\prime}=\frac{\partial}{\partial \Theta}\left(J_{21} f_{1,1}+J_{22} f_{1,2}+J_{23} f_{1,3}+J_{24} f_{1,4}\right)
\end{aligned}
$$

By rearranging eqs. ( 76 ), ( 77 ), ( 78 ), ( 79 ), and ( 94 ), the following relation is finally obtained.

$$
\begin{aligned}
& {\left[\begin{array}{c}
\dot{v}_{c} \\
\dot{\alpha}_{b} \\
\dot{q} \\
\dot{\theta} \\
\ddot{\alpha_{b}}
\end{array}\right]=\left[\begin{array}{cc}
J_{11} & J_{12} \\
J_{21} & J_{22} \\
J_{31}-\left(J_{21}{ }^{2}+2 \xi_{\alpha} \omega_{\alpha} J_{21}\right) P_{\delta_{e}} & J_{32}-\left(J_{22}{ }^{2}+2 \xi_{\alpha} \omega_{\alpha} J_{22}+\omega_{\alpha}{ }^{2}\right) P_{\delta_{e}} \\
J_{41} & J_{42} \\
\frac{\partial L_{F_{1}}{ }^{2} h_{1}(\alpha)}{\partial V_{c}}\left(1-P_{\delta_{e}}\right)-\left(2 \xi_{\alpha} \omega_{\alpha} J_{21}\right) P_{\delta_{e}} & \frac{\partial L_{F_{1}}{ }^{2} h_{1}(\alpha)}{\partial \alpha}\left(1-P_{\delta_{e}}\right)-\left(2 \xi_{\alpha} \omega_{\alpha} J_{22}+\omega_{\alpha}{ }^{2}\right) P_{\delta_{e}}
\end{array}\right.}
\end{aligned}
$$

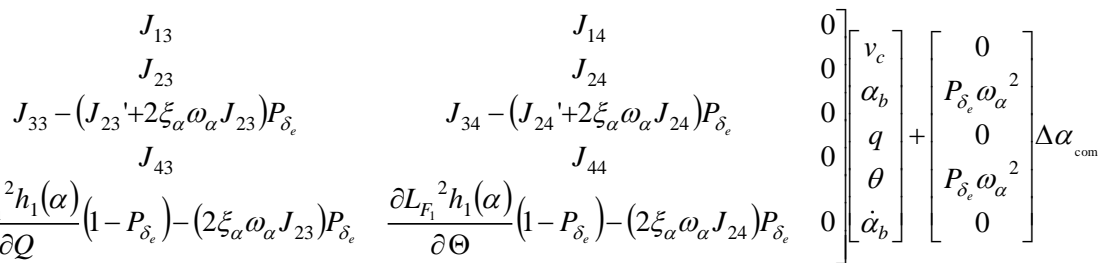

Because this system has rank 4, and the second-order derivative of angle of attack is independent, it can be omitted as follows.

$$
\begin{aligned}
\dot{\mathbf{x}} & =A \mathbf{x}+B u \\
y & =C \mathbf{x}
\end{aligned}
$$

where, each matrix is expressed by

$$
\begin{aligned}
& A=\left[\begin{array}{cc}
J_{11} & J_{12} \\
J_{21} & J_{22} \\
J_{31}-\left(J_{21}{ }^{\prime}+2 \xi_{\alpha} \omega_{\alpha} J_{21}\right) P_{\delta_{e}} & J_{32}-\left(J_{22}{ }^{\prime}+2 \xi_{\alpha} \omega_{\alpha} J_{22}+\omega_{\alpha}{ }^{2}\right) P_{\delta_{e}} \\
J_{41} & J_{42}
\end{array}\right. \\
& \left.\begin{array}{cc}
J_{13} & J_{14} \\
J_{23} & J_{24} \\
J_{33}-\left(J_{23}{ }^{\prime}+2 \xi_{\alpha} \omega_{\alpha} J_{23}\right) P_{\delta_{e}} & J_{34}-\left(J_{24}{ }^{\prime}+2 \xi_{\alpha} \omega_{\alpha} J_{24}\right) P_{\delta_{e}} \\
J_{42} & J_{44}
\end{array}\right] \\
& B=\left[\begin{array}{c}
0 \\
0 \\
P_{\delta_{e}} \omega_{\alpha}^{2} \\
0
\end{array}\right] \\
& C=\left[\begin{array}{l}
0 \\
1 \\
0 \\
0
\end{array}\right]^{T}
\end{aligned}
$$

Finally, the transfer function of linearized hierarchal dynamic inversion is expressed as follows.

$$
G_{\text {lon }}=C(s I-A)^{-1} B
$$


Hereafter, this transfer function is called "linearization".

\section{Linearized Analysis and Simulations:-}

When the control performance is evaluated, the pseudo-input generated by eq. ( 53 ) must be used. By utilizing PD control gains in eq. ( 53 ) instead of $\omega_{\alpha}$ and $\zeta_{\alpha}$, pseudo-input is expressed as follows.

$$
v_{\alpha}=K_{\alpha}\left(\alpha_{\text {com }}-\alpha\right)-K_{\dot{\alpha}} \dot{\alpha}
$$

where proportional gain is $K_{\alpha}=\omega_{\alpha}^{2}$, and derivative gain is $K_{\dot{\alpha}}=2 \zeta_{\alpha} \omega_{\alpha}$.

In this section, $\omega_{\alpha}=1 \mathrm{~Hz}, \zeta_{\alpha}=1 / \sqrt{2}$ are constant, and the natural frequency of actuator, $\omega_{\delta e}$ is varied from $1 \mathrm{~Hz}$ to $10 \mathrm{~Hz}$ with the increment of $0.5 \mathrm{~Hz}$. Damping coefficient of actuator is fixed as $\zeta_{\delta e}=1 / \sqrt{2}$. Figure 3 shows the eigenvalues of the transfer functions of eq. ( 54 ) and eq. ( 55 ).

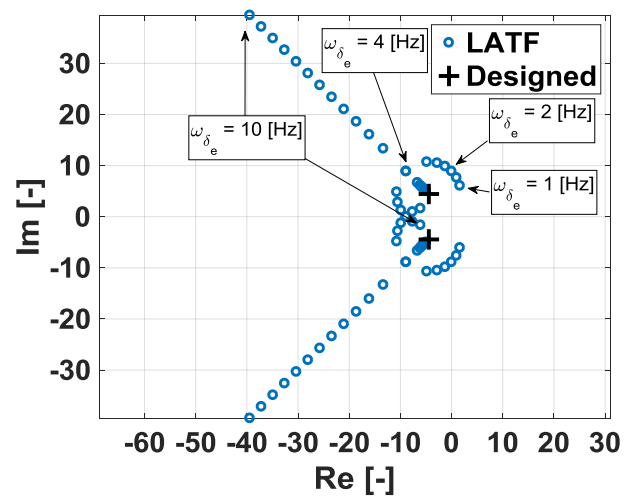

Figure 3. Eigenvalue of LATF and its designed value

Based on the eigenvalue of LATF, when damping coefficients are $\zeta_{\delta e}=1 / \sqrt{2}$ and $\zeta_{\alpha}=1 / \sqrt{2}$, the natural frequency of the actuator have to be more than twice the designed frequency of angle of attack, that is, $\omega_{\delta e} \geq 2 \mathrm{~Hz}$. In addition, when the natural frequency of the actuator is more than four times the designed frequency of angle of attack, the damping coefficient becomes the same as the designed value of $1 / \sqrt{2}$. Finally, the eigenvalues of LATF approaches to the designed frequency of angle of attack as increasing the natural frequency of the actuator.

The numerical simulation was carried out in order to compare the time response of LATF and that of the original plant dynamics. The altitude is fixed at $5,000 \mathrm{~m}$, and initial value of angle of attack $\alpha$ is $5^{\circ}$. The other status was derived according to the following steps.

Step 1) It is assumed that the status is equilibrium, and eq. ( 36 ) is satisfied. As a consequence, the following equations result from $\dot{V}_{c}=0 \dot{\alpha}=0$, and $Q=0$.

$$
\begin{aligned}
& \Theta\left(t_{0}\right)=\tan ^{-1}\left\{\frac{-C_{D}\left(\alpha\left(t_{0}\right)\right)}{C_{L}\left(\alpha\left(t_{0}\right)\right)}\right\}+\alpha\left(t_{0}\right) \\
& V_{c}\left(t_{0}\right)=\sqrt{\frac{2 m g}{\rho S C_{L}\left(\alpha\left(t_{0}\right)\right)} \cos \left(\Theta\left(t_{0}\right)-\alpha\left(t_{0}\right)\right)}
\end{aligned}
$$

Step 2) From $\dot{Q}=f_{1,3}\left(\mathbf{x}_{1}\right)+g_{1,3}\left(\mathbf{x}_{1}\right) \delta_{e}=0$, initial elevator angle is expressed as follows.

$$
\delta_{e}\left(t_{0}\right)=-\frac{C_{m}\left(\alpha\left(t_{0}\right)\right)}{C_{m_{\delta_{e}}}}
$$

Simulations were carried out with the five values of $\omega_{\delta_{e}}=1,2,3,4,10 \mathrm{~Hz}$. Each of them corresponds to the following situation.

- When $\omega_{\delta e}=1 \mathrm{~Hz}$, LATF is not stable.

- When $\omega_{\delta e}=2 \mathrm{~Hz}, \mathrm{LATF}$ is at the stability limit.

- When $\omega_{\delta e}=3 \mathrm{~Hz}$, the natural frequency of the actuator is less than 4 times the designed frequency of angle of attack, and the damping coefficient of LATF differs from that of the designed value. 
- When $\omega_{\delta e}=4 \mathrm{~Hz}$, the natural frequency of the actuator is just 4 times the designed frequency of angle of attack, and all the eigenvalues of LATF have the identical value.

- When $\omega_{\delta e}=10 \mathrm{~Hz}$, the natural frequency of the actuator is more than 4 times the designed frequency of angle of attack.

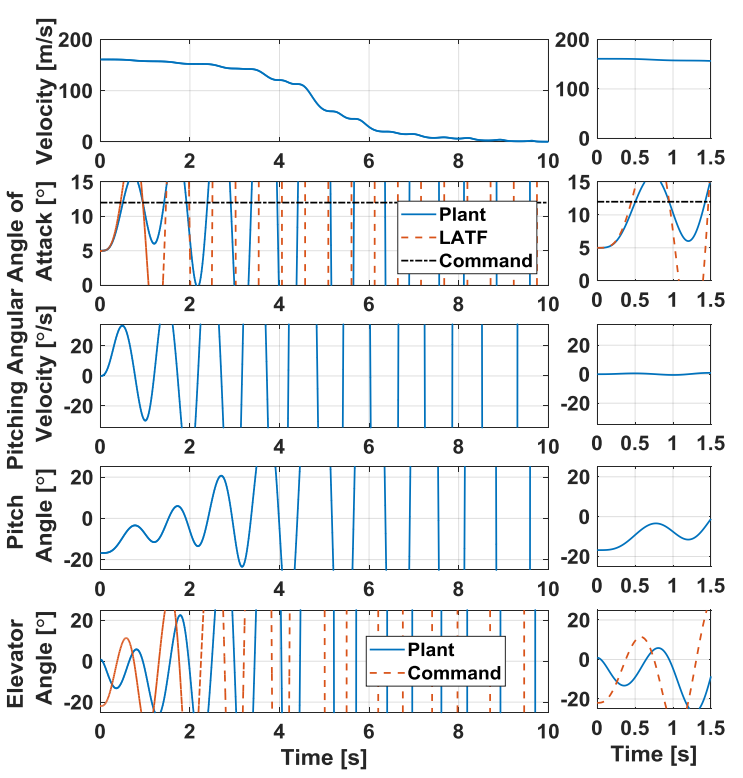

Figure 4:- Time response when $\omega_{\delta_{e}}=1 \mathrm{~Hz}$

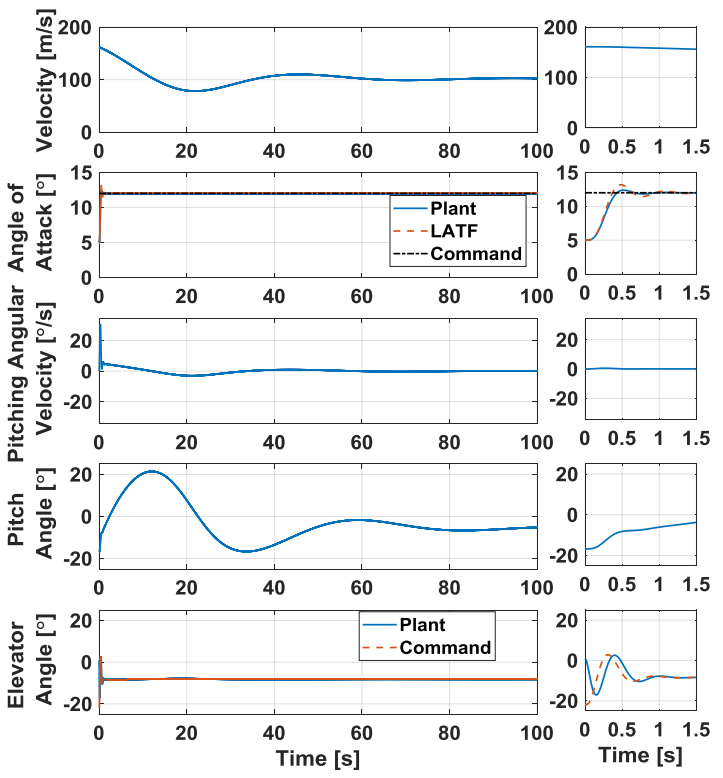

Figure 6:- Time response when $\omega_{\delta_{e}}=3 \mathrm{~Hz}$
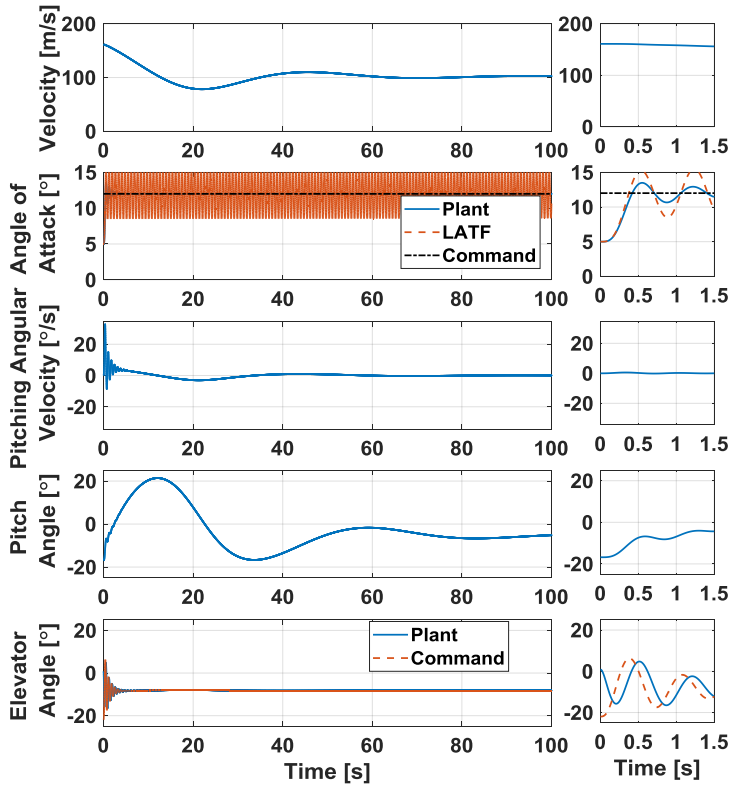

Figure 5:- Time response when $\omega_{\delta_{e}}=2 \mathrm{~Hz}$
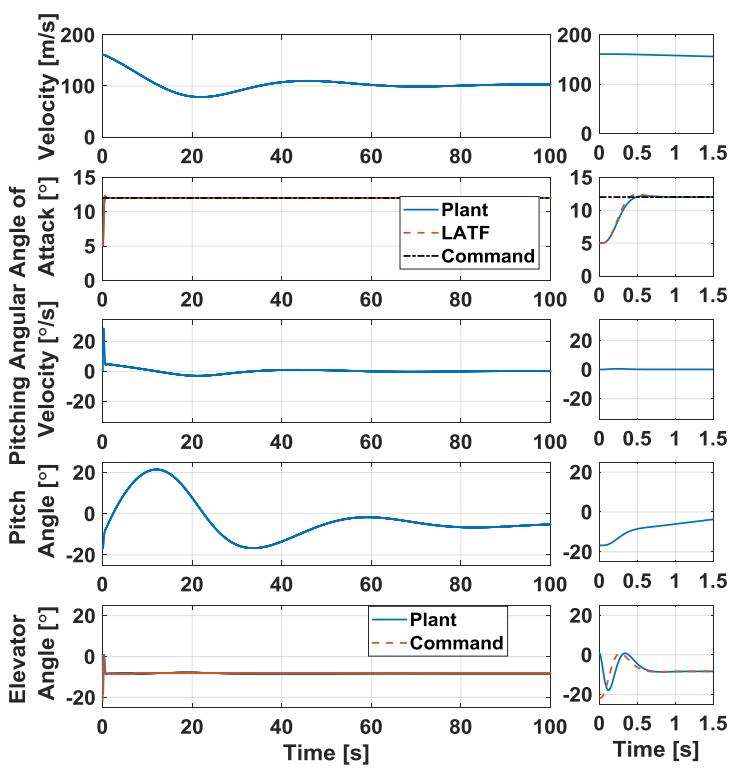

Figure 7:- Time response when $\omega_{\delta_{e}}=4 \mathrm{~Hz}$ 


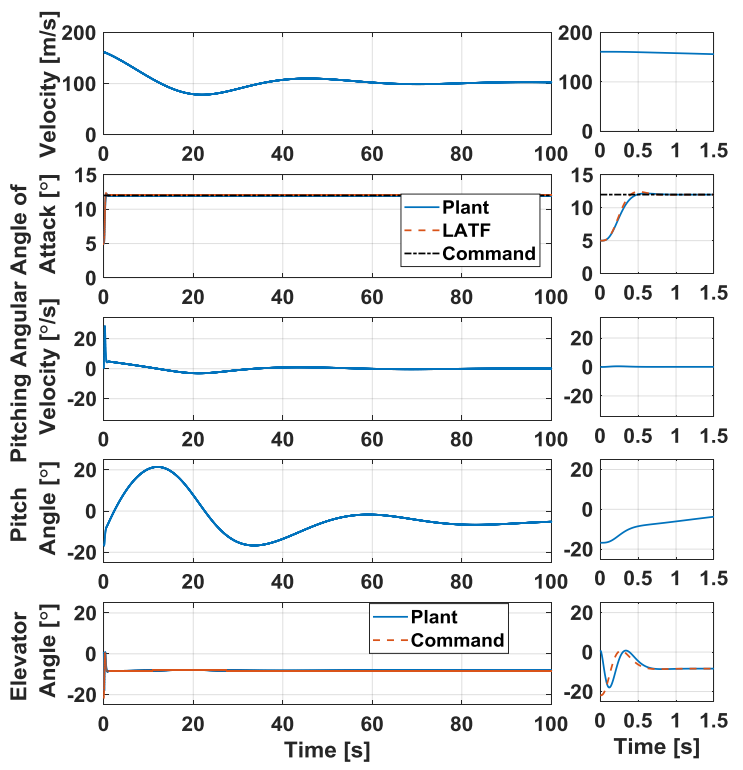

Figure 8:- Time response when $\omega_{\delta_{e}}=10 \mathrm{~Hz}$

Eigenvalue analysis of LATF in the case of $\omega_{\delta e}=1 \mathrm{~Hz}$ shown in Fig.3 is consistent with the diverging response observed in Fig.4. The case of $\omega_{\delta e}=2 \mathrm{~Hz}$ is at the stability limit based on the eigenvalue analysis of LATF, and the simulation result indicates that the plant has asymptotically converging characteristics. The reason of this difference can be attributed to the dynamic stability of nonlinear terms ignored in LATF. In the remaining cases, the eigenvalue analysis means that LATF are stable. The simulation results when $\omega_{\delta e}=3,4$, and $10 \mathrm{~Hz}$ are presented in Figs. 7, 8, and 9, respectively, and they show that the angle of attack converges from initial value to command value. In addition, since there is no significant difference between the time response of LATF and that of the plant, the influence of nonlinear terms in eq. ( 49 ) is small.

In order to evaluate the effects of nonlinear dynamics in the frequency domain, the transfer function derived from eq. ( 97 ) is employed. As a comparison, the desired eigenvalue from the transfer function of eq. ( 55 ), the eigenvalue of LATF from eq. ( 54 ), and the eigenvalue from the instantaneous linearization of the vehicle status are prepared. Note that the result of longitudinal motion simulation is utilized as the vehicle status.

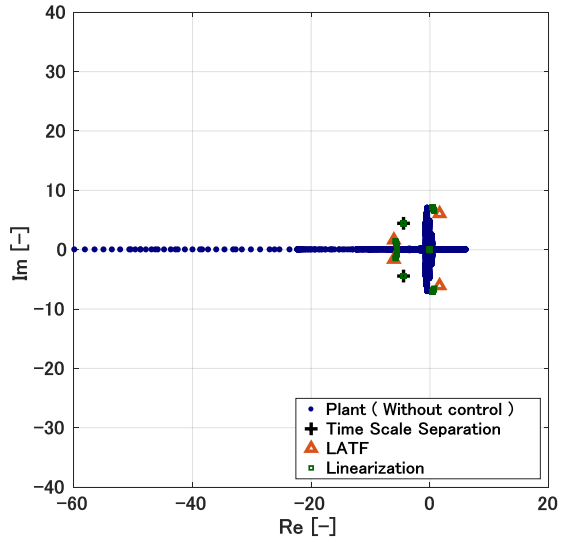

Figure 9. Comparison of eigenvalues when $\omega_{\delta_{e}}=1 \mathrm{~Hz}$

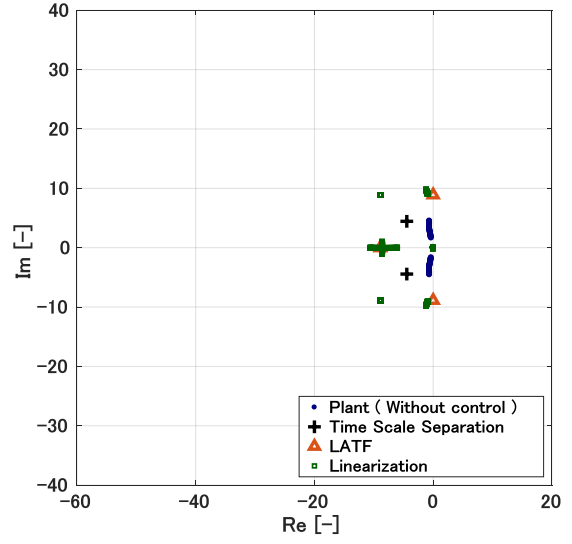

Figure 10. Comparison of eigenvalues when $\omega_{\delta_{e}}=2 \mathrm{~Hz}$ 


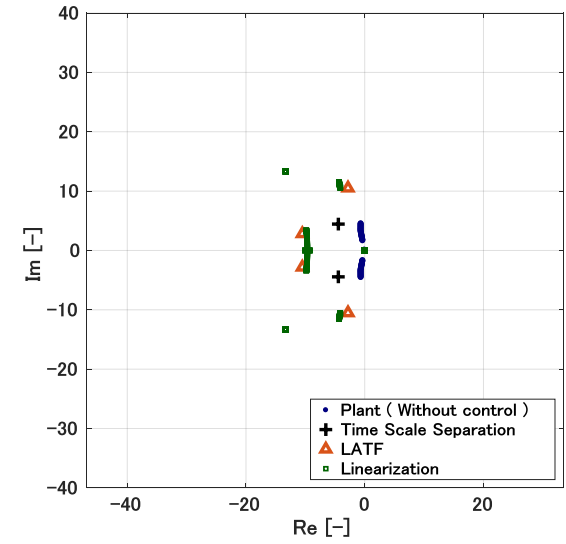

Figure 11. Comparison of eigenvalues when $\omega_{\delta_{e}}=3 \mathrm{~Hz}$

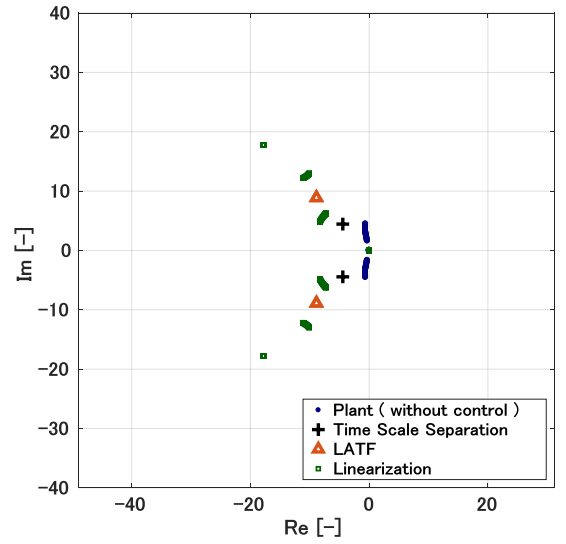

Figure 12. Comparison of eigenvalues when $\omega_{\delta_{e}}=4 \mathrm{~Hz}$

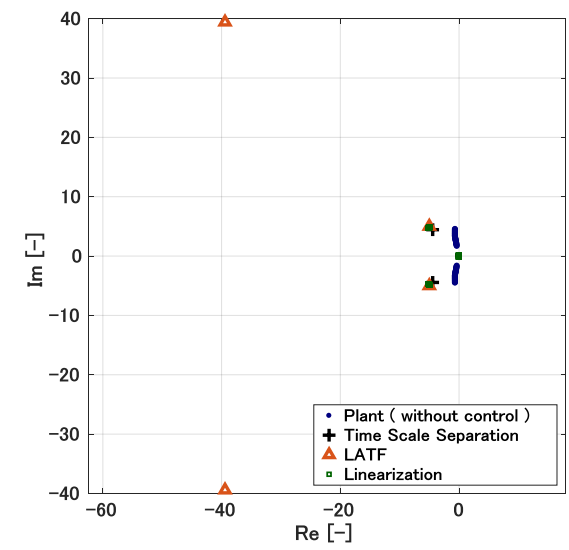

Figure 13. Comparison of eigenvalues when $\omega_{\delta_{e}}=10 \mathrm{~Hz}$

\section{Discussions:-}

Figure 9-Figure 13 show the eigenvalues with different natural frequencies of the actuator. The eigenvalue obtained in the time scale separation means the designed value, since it is assumed that the actuator response does not have delay in the time scale separation. In addition, the eigenvalue obtained in LATF is constant in each case, since it does not depend on the nonlinear vehicle dynamics. On the other hand, the eigenvalue of the plant without control and that of linearization are not constant, since they are influenced by nonlinear dynamics. The following four observations can be found from this analysis.

1. Linearization tends to be similar to LATF, which is especially apparent when $\omega_{\delta e}=2$ and $3 \mathrm{~Hz}$.

2. When the natural frequency of the actuator becomes higher than the above value, the eigenvalues of linearization tend to asymptotically converge the desired eigenvalue.

3. When LATF is at the stability limit, namely $\omega_{\delta e}=2 \mathrm{~Hz}$, all the eigenvalues resulting from linearization are negative values, and the angle of attack response of the plant model shown in Fig. 5 converged asymptotically.

4. As the natural frequency of the actuator increases, the influence of nonlinear dynamics tends to decrease, and the fluctuation range of linearization eigenvalue gets smaller.

The LATF in the hierarchal dynamic inversion method is constructed based on the assumption that the influence of the nonlinear term is small. This can be confirmed by the fact that the difference between the eigenvalues of linearization and LATF are smaller than those of linearization and time scale separation. Since LATF can be built independently from the plant model as shown in eq. ( 54 ), LATF can be conveniently utilized as an initial design guideline of the control law. In the practical application where the influence of plant model dependency must be evaluated, the eigenvalue analysis based on the time series data obtained from the flight trajectory ( eq. ( 97 ), ( 98 ) ) 
is useful. Although it is difficult to prevent instability completely using this method, it is possible to evaluate the instability in finite states using such eigenvalue analysis.

Based on these discussions, two notable findings are obtained. First, LATF has the response characteristics that resembles the plant, especially when the response of the actuator becomes faster than the designed frequency. Second, the exact input-output linearization cannot be performed using the control law designed by the hierarchal dynamic inversion methodology in contrast to pure dynamic inversion theory. Therefore, the response of the designed feedback system is influenced by dynamic characteristic of the plant. Therefore, linearized eigenvalue analysis should be conducted utilizing the simulated flight trajectory.

\section{Conclusions:-}

This paper presented the methodology for the stability analysis of a control law based on hierarchical dynamic inversion considering the application to a winged rocket. A novel concept named Linearized Approximation Transfer Function (LATF) is derived by omitting nonlinear term caused by the lower hierarchy (a second-order delay element of the actuator for aerodynamic control surfaces). LATF is determined only by the actuator characteristics and pseudo input, and it does not depend on the vehicle dynamics. Therefore, the preliminary performance of the designed control law can be evaluated conveniently using LATF. On the other hand, since LATF ignores the influence of nonlinear term exactly, the evaluation including the nonlinear term is indispensable. In this paper, eigenvalues of an instantaneous linearization based on the time series data are analyzed. As a result, the effect of nonlinear term in controlled variable is smaller than that of linear term, and the influence of nonlinear dynamics tends to decrease as the natural frequency of the actuator increases. The above eigenvalue analysis also clarifies the natural frequency difference between the vehicle and actuator required for the stabilization, which has been impossible using previous dynamic inversion techniques. In addition, the time response of plant is found to be almost same as that of LATF.

\section{References:-}

1. A. Isidori, Nonlinear Control Systems, Springer-Verlag, Berlin, Heidelberg, (1995), pp.145-172

2. Y. Ochi, and K. Kanai, 'Design of Restructurable Flight Control Systems Using Feedback Linearization,' Journal of Guidance, Control, and Dynamics, 14-5 (1991), pp.903-911

3. G. Tao, 'Adaptive Control Design and Analysis,' John Wiley \& Sons, (2003), pp.535-541,

4. P. K. A. Menon, M.E. Badgett, R.A. Walker, and E.L. Duke, 'Nonlinear Flight Test Trajectory Controllers for Aircraft,' Journal of Guidance, Control, and Dynamics, 10-1 (1987), pp.67-72

5. P. K. A. Menon, M. Yousefpor, 'Design of Nonlinear Autopilots for High Angle of Attack Missiles,' Proceeding of the AIAA Guidance, Navigation, and Control Conference, AIAA, Palo Alto, California, (1996), pp.29-31

6. P. K. A. Menon, V.R. Iragavarapu, E.J. Ohlmeyer, 'Nonlinear Missile Autopilot Design Using Time-Scale Separation,' Proceeding of the AIAA Guidance, Navigation, and Control Conference, AIAA, New Orleans, Los Angles, (1997), pp.1791-1803

7. P. K. A. Menon, E. J. Ohlmeyer, 'Computer-Aided Synthesis of Nonlinear Autopilots for Missiles,' Optimal Synthesis, 11-2 (2004), pp.173-198

8. D. Ito, J. Georgie, J. Valasek, D. T. Ward, et al, 'Reentry Vehicle Flight Controls Design Guidelines: Dynamic Inversion,' NASA/TP-2002-210771 (2002)

9. S. Sunasawa, and H. Ohta, 'Nonlinear Flight Control for a Reentry Vehicle Using Inverse Dynamics Transformation,' Journal of the Japan Society for Aeronautical and Space Sciences, 45-516 (1997), pp.52-61 (in Japanese)

10. J. Kawaguchi, Y. Miyazawa, and T. Ninomiya, 'Flight Control Law Design with Hierarchy-Structured Dynamic Inversion Approach,' Proceeding of the AIAA Guidance, Navigation, and Control Conference, AIAA, 2008-6959, Honolulu, Hawaii, (2008)

11. T. Ninomiya, H. Suzuki, and J. Kawaguchi, 'Evaluation of Guidance and Control System of D-SEND\#2,' IFAC-PapersOnline, 49-17 (2016), pp.106-111

12. T. Shimozawa, T. Narumi, S. Sagara, and K. Yonemoto, 'Digital Adaptive Control of Winged Rocket Using Unscented Kalman Filter,' Artificial Life and Robotics, 16-3 (2011), pp.348-351

13. D. S. Naidu, and A. J. Calise, 'Singular Perturbations and Time Scales in Guidance and Control of Aerospace Systems: A Survey,' Journal of Guidance, Control, and Dynamics 24-6 (2001), pp.1057-1078 
14. C. J. Schumacher, et al. 'Stability Analysis of Dynamic Inversion Controllers Using Time-Scale Separation,' Proceeding of the Guidance, Navigation, and Control Conference and Exhibit, 98-4322 (1998), pp.1217-1223

15. P. V. Kokotovic, et al. Singular Perturbation Methods in Control Analysis and Design, Society for Industrial and Applied Mathematics, (1999), pp.17-40

16. A. Abe, and Y. Shimada, 'Flight Control System Using Backstepping Method for Space Transportation System,' Transactions of the Japan Society for Aeronautical and Space Science, Aerospace technology Japan, 10-ists28 (2012), Pd_85-Pd_91

17. A. Abe, K. Iwamoto, and Y. Shimada, 'Design of Flight Control System Based on Adaptive Backstepping Method for a Space Transportation System,' Transactions of the Japan Society for Aeronautical and Space Sciences, Aerospace technology Japan, 58-2 (2015), pp.55-65

18. M. Krstic, L. Kanellakopouios, and P. V. Kokotovic, 'Nonlinear and Adaptive Control Design,'WileyInterscience (1995), pp.64-66

19. M. Wakita, K. Yonemoto, et al. ,'Preliminary Design of Winged Experimental Rocket by University Consortium,' Transactions of the Japan Society for Aeronautical and Space Science, Space Technology Japan, 7-ists26(2009), Tg_21-Tg_26

20. G. S. Gossamsetti, et al, 'Recent Developments of Experimental Winged Rocket: Autonomous Guidance and Control Demonstration Using Parafoil, Procedia Engineering, 99 (2015), pp.156-162

21. K. Itakura, et al., 'Development and Ground Combustion Test of a Subscale Reusable Winged Rocket,' Transactions of the Society for Aeronautical and Space Sciences, Aerospace Technology Japan, 12-ists29 (2014), To_3_1/To_3_5

22. T. Ohki, et al., 'Recent Developments of Experimental Winged Rocket: Autonomous Guidance and Control Demonstration by Flight Test,' Proceeding of the Asia-Pacific International Symposium on Aerospace Technology, Cairns, Australia, (2015), pp.408-414

23. K. Yonemoto, T. Fujikawa, et al, 'Recent Flight Test of Experimental Winged Rocket and Its Future Plan for Suborbital Technology Demonstration,' Proceeding of the International Astronautical Congress, Guadalajara, Mexico, (2016), pp. IAC-16-D2.6.1 